BNL-114115-2017-JA

\title{
Fast Holes, Slow Electrons, and Medium Control of Polaron Size and Mobility in the DA Polymer F8BT
}

\author{
M. J. Bird, J. Bakalis, S. Asaoka, H. Sirringhaus, and J. R. Miller
}

Submitted to Journal of Physical Chemistry C

June 2017

Chemistry Department

Brookhaven National Laboratory

\author{
U.S. Department of Energy \\ USDOE Office of Science (SC), \\ Basic Energy Sciences (BES) (SC-22)
}

Notice: This manuscript has been authored by employees of Brookhaven Science Associates, LLC under Contract No. DE- SC0012704 with the U.S. Department of Energy. The publisher by accepting the manuscript for publication acknowledges that the United States Government retains a non-exclusive, paid-up, irrevocable, world-wide license to publish or reproduce the published form of this manuscript, or allow others to do so, for United States Government purposes. 


\section{DISCLAIMER}

This report was prepared as an account of work sponsored by an agency of the United States Government. Neither the United States Government nor any agency thereof, nor any of their employees, nor any of their contractors, subcontractors, or their employees, makes any warranty, express or implied, or assumes any legal liability or responsibility for the accuracy, completeness, or any third party's use or the results of such use of any information, apparatus, product, or process disclosed, or represents that its use would not infringe privately owned rights. Reference herein to any specific commercial product, process, or service by trade name, trademark, manufacturer, or otherwise, does not necessarily constitute or imply its endorsement, recommendation, or favoring by the United States Government or any agency thereof or its contractors or subcontractors. The views and opinions of authors expressed herein do not necessarily state or reflect those of the United States Government or any agency thereof. 


\section{Fast Holes, Slow Electrons and Medium Control of}

\section{Polaron Size and Mobility in the DA Polymer}

\section{F8BT}

Matthew J. Bird ${ }^{\dagger *}$, Jin Bakalis ${ }^{\dagger}$, Sadayuki Asaoka ${ }^{\S}$,Henning Sirringhaus ${ }^{\circ}$, and John R. Miller $^{\dagger}$

${ }^{\dagger}$ Chemistry Department, Brookhaven National Laboratory, Upton, New York 11973, USA

${ }^{\S}$ Department of Biomolecular Engineering, Kyoto Institute of Technology, Matsugaskaki, Sakyo-ku, Kyoto 606-8585, Japan

${ }^{\circ}$ Cavendish Laboratory, University of Cambridge, J J Thomson Avenue, Cambridge, CB3 OHE, UK 


\section{ABSTRACT}

The nature of electron and hole polarons on poly(9,9-di-n-hexylfluorenyl-2,7-diyl) (pF) and a copolymer poly[(9,9-di-n-octylfluorenyl-2,7-diyl)-alt-(benzo[2,1,3]thiadiazol-4,8-diyl)] (F8BT) has been studied by chemical doping, pulse radiolysis, charge modulation spectroscopy, quantum chemical calculations and microwave conductivity. While pF exhibits very similar behavior in all respects for the electron and the hole, this paper explores the hypothesis that the donor acceptor (push-pull) nature of F8BT will tend to localize charges. Optical spectra and quantum chemical calculations point to an electron localized on the thiadiazole unit in polar liquids, but becoming more delocalized as the solvent polarity decreases. Indeed, in the non-polar solvent benzene, the electron mobility is only 2.7 times lower than that of the hole, which conversely is shown to be delocalized in all environments and has a similar mobility to polarons on the homopolymer polyfluorene. Advantageous modifications to the optoelectronic properties of conjugated polymers, that come about by using alternating donor acceptor repeat units, have thus been shown to not significantly hinder charge transport despite the corrugated energy landscape along the backbone. 


\section{INTRODUCTION}

“Push-pull” or donor-acceptor (DA) conjugated polymers, consisting of alternating donoracceptor repeat units, have become potent in the field of organic electronics. ${ }^{1}$ DA polymers are important to the possibility of organic photovoltaics (OPV) because they reduce the energy gap between the highest occupied and lowest unoccupied molecular orbitals (HOMO and LUMO) to achieve a better absorption match to the solar spectrum and give new paths by which chemists can elaborate the structures to achieve aims of OPV and organic electronics. They often also have large electron affinities, giving air-stable electron transport, which is advantageous in OPVs and field effect transistors (OFETs) respectively. On the other hand delocalization and transport may be reduced for charges that move in the corrugated potentials of DA polymers, in which electrons might localize on the acceptor groups and holes on donor groups, thus producing more localized and potentially slow moving polarons.

Relatively little is known about the nature of polarons in push-pull polymers. In this paper we do a thorough comparison between the homopolymer poly(9,9-di-n-hexylfluorenyl2,7-diyl) (pF) and a DA copolymer, poly[(9,9-di-n-octylfluorenyl-2,7-diyl)-alt(benzo[2,1,3]thiadiazol-4,8-diyl)] (F8BT) to address the effect that the corrugated energy landscape of the backbone has on polaron localization, optical absorptions and intrachain mobility. Data presented here will examine the nature of polarons in F8BT, both with and without counter ions, in non-polar surroundings and moderately polar surroundings that might enhance localization, and will measure mobilities in a non-polar medium.

Experiments described below will determine delocalization lengths of isolated electrons and holes in F8BT and compare them with our previous report ${ }^{2}$ that found electron polarons to have 4-6 nm lengths in polyfluorenes and ladder-type poly(para-phenylene).

The FBC model ${ }^{3}$ predicts two absorption peaks for polarons in their spectra, denoted as $\mathrm{P}_{1}$ and $\mathrm{P}_{2}$, in conjugated polymers. Different behavior will be found for electron polarons in F8BT. To obtain spectra of polarons, free from ion pairing, in low polarity media akin to that 
found in devices, two methods are used here: charge modulation spectroscopy (CMS) and pulse radiolysis (PR). Both methods will determine absolute absorption intensities as extinction coefficients, $\varepsilon\left(\mathrm{M}^{-1} \mathrm{~cm}^{-1}\right)$, or equivalently, cross-section, $\sigma\left(\mathrm{cm}^{2}\right)=10^{3} \times \varepsilon \ln (10) / N_{A}$.

CMS (Figure 1) is a sensitive lock-in technique for measuring the optical absorption of polarons in a transistor or metal-insulator-semiconductor (MIS) structure. ${ }^{4-6}$ CMS typically modulates the charges with frequencies in the tens of Hz. The advantages of CMS over DC methods are that it is only sensitive to mobile charges, the phase information allows artifacts like electroabsorption to be identified and removed and the modulated charge density can be simultaneously measured electrically to eliminate errors in extinction coefficient estimates caused by charge trapping. ${ }^{7}$ Typically CMS has been used to study hole polarons, ${ }^{4-6,8-9}$ as hole transporting transistors had historically been easier to fabricate owing to electron trapping and injection issues. ${ }^{10}$ More recently, electron accumulation has been used to study negative polarons in $\mathrm{N} 2200^{11}$ and both holes and electrons in homopolymer polyselenophene, ${ }^{12}$ and in donor-acceptor (DA) polymers using both $\mathrm{CMS}^{7}$ and a DC bias method. ${ }^{13}$ An important artifact to be aware of in these types of measurements arises if the detector picks up fluorescence from the, often highly luminescent, polymer which can be modulated by a very efficient exciton-polaron quenching mechanism, however this can be corrected with the use of an appropriate filter. ${ }^{7}$

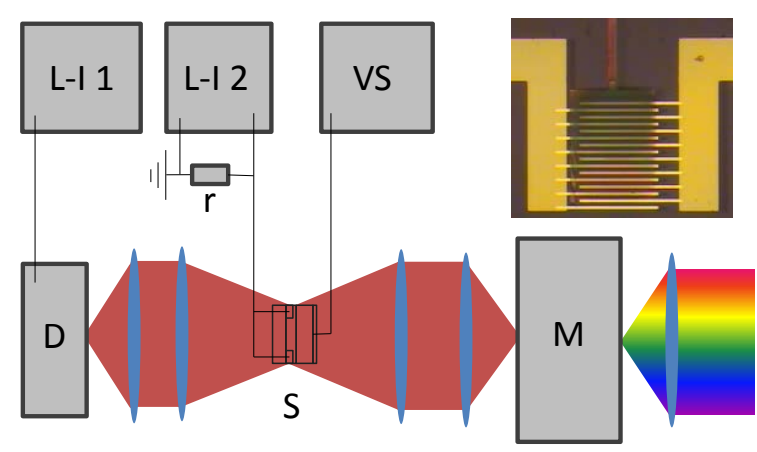

Figure 1. Charge Modulation Spectroscopy apparatus showing monochromator (M), detector (D) two lock in amplifiers (L-I 1 and L-I 2), OFET sample (S) and AC voltage source (VS). Inset shows optical micrograph of the transistor with PEDOT gate electrode. 
Pulse radiolysis uses a high energy, short ( $<50 \mathrm{ps})$ electron pulse to rapidly inject charges, of the desired polarity and a known concentration, onto polymer chains in many different solvents. ${ }^{14}$ If the polaron finds a counter-ion it will typically annihilate with it, meaning that the obtained spectra are due to free ions, unpaired with counter-ions.

Microwave conductivity with pulse radiolysis has been used to study intramolecular charge transport, most commonly that of holes, in a number of polymers, including polyfluorene where the first reported $1 \mathrm{D}$ intramolecular mobility was $0.74 \mathrm{~cm}^{2} / \mathrm{Vs}{ }^{15}$ Comparisons of the electron and hole microwave mobility along a single chain are also limited to a few techniques and to the authors knowledge have only been performed on a limited number of polymers using microwave conductivity. The $1 \mathrm{D}$ mobility, related to the measured isotropic mobility $\mu_{\text {iso }}$ by $\mu_{1 \mathrm{D}}=3 \times \mu_{\text {iso }}$, was found to be $0.5 \mathrm{~cm}^{2} / \mathrm{Vs}$ and $0.2 \mathrm{~cm}^{2} / \mathrm{Vs}$ for electrons and holes respectively on PPV in benzene using $\mathrm{NH}_{3}$ and $\mathrm{CCl}_{4}$ as hole and electron scavengers. ${ }^{16}$ A lower limit of $\mu_{1 \mathrm{D}}=0.5 \mathrm{~cm}^{2} / \mathrm{Vs}$ for both electrons and holes were found for $\mathrm{pF}$ in trans-decalin using $\mathrm{NH}_{3}$ and $\mathrm{CO}_{2}$ as hole and electron scavengers. ${ }^{17}$

Localization of the electron on the benzothiadiazole (BT) unit has been expected to reduce the electron mobility compared to the hole mobility in F8BT. ${ }^{18-19}$ However, most measurements in films indicate that, once electron traps are filled, the mobilities of electrons and holes are similar. Zaumseil found FET mobilities, $\mu_{\mathrm{FET}}$, of $2.2 \times 10^{-3} \mathrm{~cm}^{2} / \mathrm{Vs}$ and $6.3 \times 10^{-}$ ${ }^{4} \mathrm{~cm}^{2} /$ Vs for holes and electrons respectively when the F8BT chains are aligned in the direction of current flow. ${ }^{20}$ Aligned films require a thicker F8BT layer which can increase contact resistance between the electrodes and the charge transport layer in top gate structures, particularly for electrons which have a lower bulk mobility due to traps. Zhang showed that electron traps are present at a density of $\sim 0.1 \%$ of polymer repeat units (PRU), but once they are filled, an identical bulk mobility of $\mu_{\mathrm{SCLC}}=3 \times 10^{-6} \mathrm{~cm}^{2} / \mathrm{Vs}$ for electrons and holes can be found by space charge limited current measurements (SCLC), ${ }^{21}$ albeit lower than $\mu_{\mathrm{FET}}$ due to the lower charge density. ${ }^{22}$ Naber also found identical electron and hole mobilities of $1 \times 10^{-2}$ 
$\mathrm{cm}^{2} / \mathrm{Vs}$ in an FET optimized with a combination of an interfacial low-k

(polycyclohexylethylene) dielectric and a high-k dielectric (polyvinylidene fluoride-

trifluoroethylene) to achieve even higher charge density. ${ }^{23}$ However, SCLC and FET

measurements not only give differences due to different charge densities but also include the effects of contact resistance and intermolecular hops which may cover any intrinsic difference in mobility for the electron and hole along the polymer backbone. The observation of a broad spatial extent to the electroluminescence emission from the recombination zone of aligned F8BT FETs gave evidence for intramolecular transport of both electrons and holes being $>1000$ times larger than intermolecular transport. However, the asymmetry in the emission profile could be explained by a lower electron bulk mobility rather than necessarily a lower electron intramolecular mobility. ${ }^{20}$

In this paper we observe microwave mobility values that differ only by a factor of 2.7 for electrons and holes on F8BT despite the possibly much larger differences in the nature of the two species. We show, with DFT calculations ${ }^{24}$ and spectroscopic evidence, that a low dielectric constant environment may increase the delocalization of the anion, which could explain why electron mobilities are not much smaller than hole mobilities. We provide spectroscopic evidence to support this.

\section{RESULTS}

Electronic Spectra for F8BT Anions and Cations from chemical doping. Figure 2 displays UV-Vis-NIR absorption spectra of F8BT anions, chemically doped with sodium biphenyl $\left(\mathrm{Na}^{+} \mathrm{Bip}^{--}\right)$in THF solution in the presence of 4,7,13,16,21,24-Hexaoxa-1,10-diazabicyclo [8.8.8] hexacosane $\left(\mathrm{C}_{222}\right)$. $\mathrm{C}_{222}$ encapsulates $\mathrm{Na}^{+}$counter-ions reducing their contact with F8BT anions. To prevent the overlap of the spectra the titration is divided into two stages based on the behavior of the $\mathrm{P}_{2}$ anion band $(\sim 2 \mathrm{eV})$. Reduction I refers to the stage in which the $\mathrm{P}_{2}$ band grows almost linearly with the number of reducing equivalents $\mathrm{n}$ (per repeat unit) 
added to reach a maximum, while the bands of the neutral decay and disappear. In reduction II, the $\mathrm{P}_{2}$ band decreases and shifts while new transitions of doubly reduced species grow for $n>1$. The evolution of the NIR band with reducing equivalents, $n$, is different from that for the visible bands. This curious behavior will be compared to a model and computed descriptions in the Discussion. Qualitatively similar behavior is seen without $\mathrm{C}_{222}$ present (Figure S6).

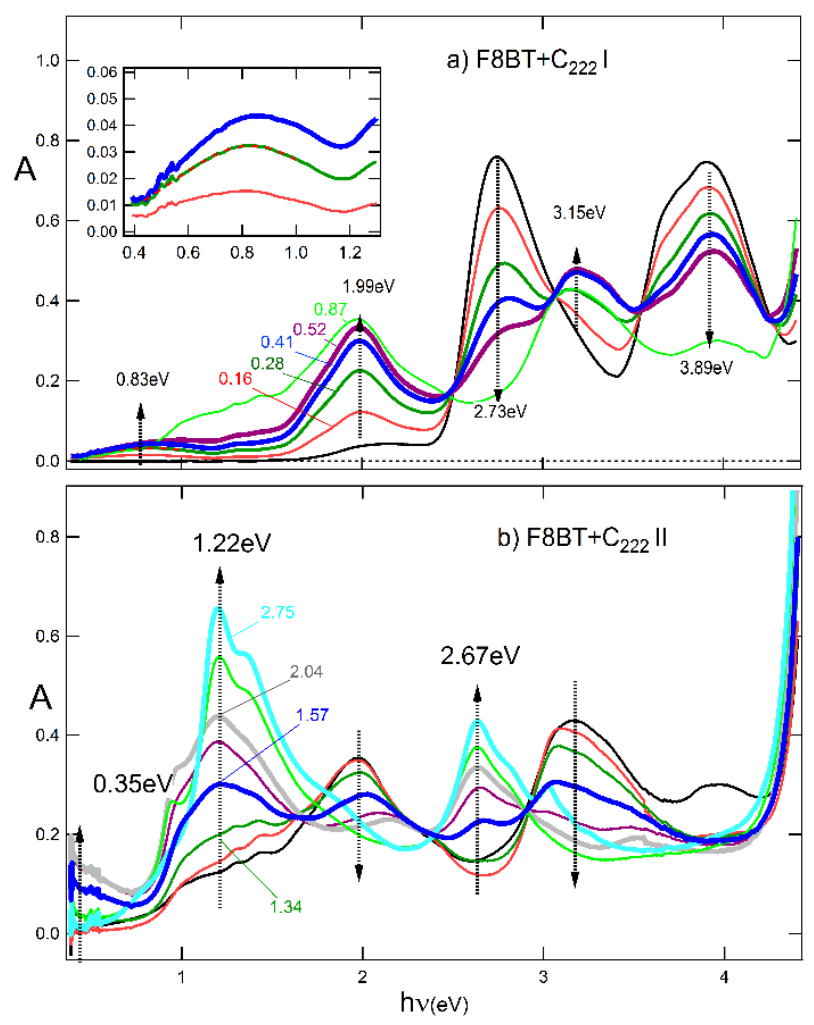

Figure 2. A series of the UV-Visible-NIR spectra of $0.33 \mathrm{mM}$ (PRU) F8BT in THF solution chemically doped with $\mathrm{Na}^{+} \mathrm{Bip}^{\bullet-}$ in the presence of $18 \mathrm{mM}$ of $\mathrm{C}_{222}$. a) reduction $\mathrm{I}$ for $\mathrm{n}=0$ 0.87 b) reduction II $(n>0.87)$. The labels are reducing equivalents $n$ as $n=$ Bip $^{\bullet-} /$ PRU.

Figure 3 displays the UV-Vis-NIR absorption spectra of F8BT $^{*+}$ produced by chemical oxidation with $\mathrm{THI}^{\circ+} \mathrm{PF}_{6}{ }^{-}$in $\mathrm{ODCB}$. $\mathrm{F} 8 \mathrm{BT}^{\circ+}$ cations have two intense bands at 0.46 and 1.73 $\mathrm{eV}$. For $\mathrm{n}>0.55$, further oxidation produced growth of the $\mathrm{THI}^{\bullet+}$ band at $2.25 \mathrm{eV}$, but little additional changes in the F8BT spectra. $\mathrm{THI}^{\bullet+} \mathrm{PF}_{6}{ }^{-}$, could not oxidize F8BT beyond $\mathrm{n}=\sim 0.3$ cations per repeat unit. The equilibrium constant for the reaction $\mathrm{F} 8 B T^{*+}+\mathrm{THI} \rightleftharpoons \mathrm{F} 8 \mathrm{BT}+$ 
$\mathrm{THI}^{\circ+}$ in $\mathrm{ODCB}$ was measured to be $1.0 \pm 0.3$ by pulse radiolysis transient absorption under conditions where there much was less than $1 \mathrm{~F} \mathrm{BT}^{\circ+}$ per chain. This equilibrium constant was applied in a model calculation shown in the inset to Figure 3 (solid line) assuming that each F8BT $^{*+}$ added to the polymer occupies 4.3 PRU as reported in Table 3. The dashed line, which assumes $\mathrm{F} 8 \mathrm{BT}{ }^{\cdot+}$ occupies 1.0 PRU does not describe well the leveling off of F8BT ${ }^{\circ+}$ growth.

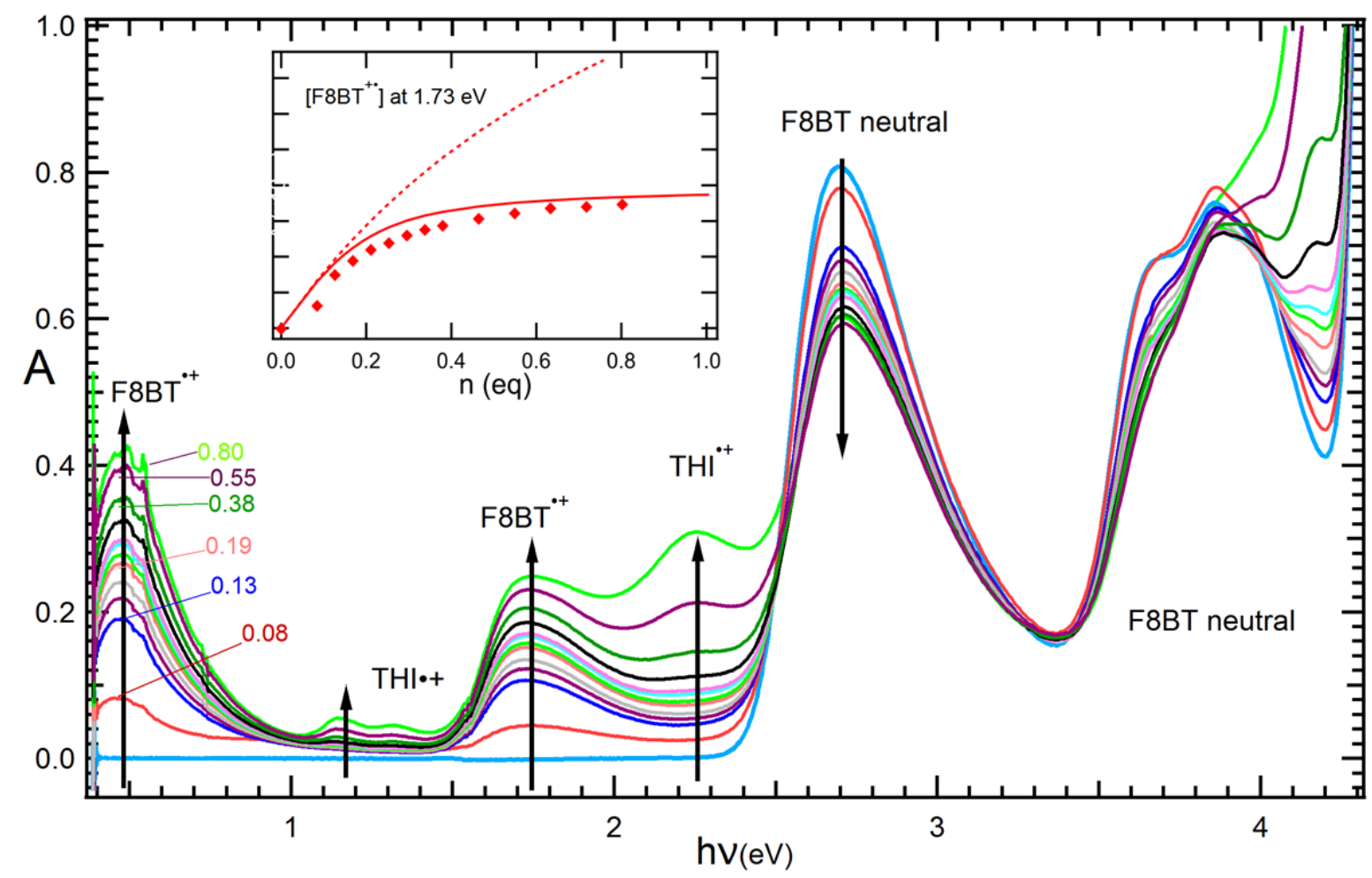

Figure 3. A series of the UV-Visible-NIR spectra of $0.34 \mathrm{mM}$ (PRU) F8BT solution in ODCB chemically doped with $\mathrm{THI}^{\bullet+} \mathrm{PF}_{6}{ }^{-}$. The labels are concentrations as $\mathrm{THI}^{{ }^{+}} / \mathrm{PRU}$. The inset plots $\mathrm{F}_{\mathrm{BBT}}{ }^{\bullet+}$ concentration ( $\left.\diamond\right)$ vs. $\mathrm{n}=\mathrm{THI}^{\bullet+} / \mathrm{PRU}$, compared with a model (-) that assumes that an $\mathrm{F}_{\mathrm{BBT}}{ }^{\bullet+}$ occupies 4.3 repeat units; the dashed line assumes each $\mathrm{F} \mathrm{BT}{ }^{\bullet+}$ occupies 1.0 repeat units.

Spectra of polarons in different environments. Figure 4 shows absorption spectra for anions and cations of $\mathrm{pF}$ and $\mathrm{F} 8 \mathrm{BT}$ in a range of different environments, normalized to the peak of the $\mathrm{P}_{2}$ absorption for clarity; extinction coefficients are reported in Table 1. 

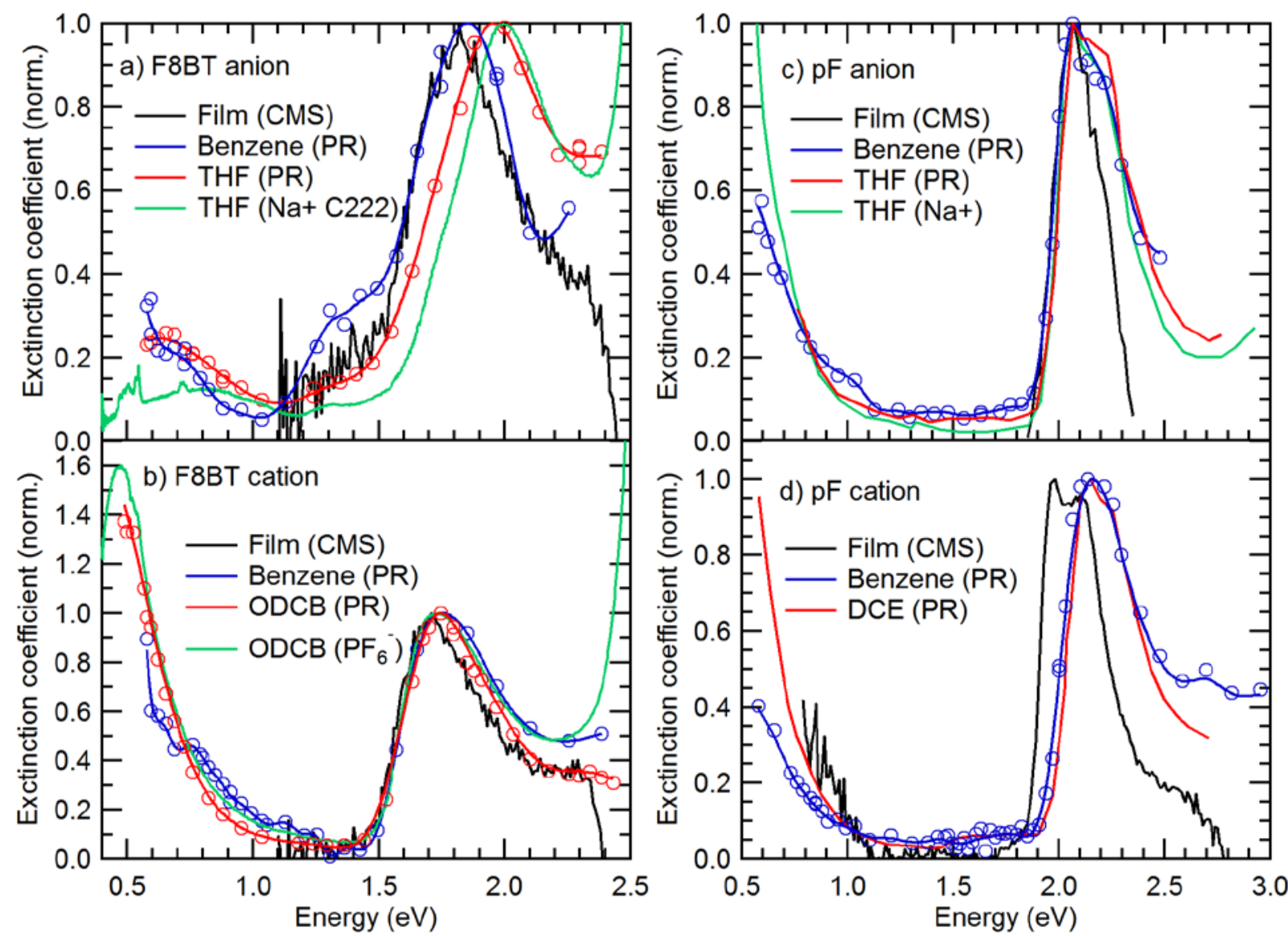

Figure 4. Spectra of cations and anions of $\mathrm{pF}$ and F8BT, normalized to the $\mathrm{P}_{2}$ absorption, taken with charge modulation spectroscopy (CMS) in films, pulse radiolysis (PR) or chemical doping in solution, with the counter-ion shown in parenthesis. Spectra in THF in c) and in DCE in d) are reproduced with permission. ${ }^{14}$ Copyright 2006 American Chemical Society.

Table 1. Extinction coefficients of anions/cations of $\mathrm{pF}$ and F8BT measured with charge modulation spectroscopy (CMS), pulse radiolysis (PR) or chemical doping in solution.

\begin{tabular}{|c|c|c|c|}
\hline Species & $\begin{array}{l}\lambda_{\max } \\
{[\mathrm{nm}]}\end{array}$ & $\begin{array}{c}\varepsilon \\
{\left[\mathrm{M}^{-1} \mathrm{~cm}^{-1}\right]}\end{array}$ & Method \\
\hline F8BT $^{-*}$ & 680 & $24,000 \pm 5,000$ & PR in benzene, benzophenone ${ }^{\mathrm{a})}$ \\
\hline F8BT** & 620 & $26,000 \pm 2,600$ & Chemical reduction in THF with NaBP \\
\hline F8BT+* & 600 & $30,000 \pm 6,000$ & PR in THF biphenyl and benzophenone ${ }^{a)}$ \\
\hline F8BT** & 674 & $30,000 \pm 3,000$ & CMS (film) \\
\hline $\mathrm{F}^{8} \mathrm{BT}^{+\bullet}$ & 710 & $42,000 \pm 8,000$ & $\mathrm{PR}$ in benzene with $\mathrm{pF}^{\mathrm{a})}$ \\
\hline $\mathrm{F} 8 \mathrm{BT}^{+}$ & 710 & $47,000 \pm 19,000$ & Chemical oxidation in ODCB with $\mathrm{THI}{ }^{\bullet+}$ \\
\hline $\mathrm{F} 8 \mathrm{BT}^{+\bullet}$ & 710 & $55,000 \pm 10,000$ & $P R$ in ODCB thianthrene ${ }^{a)}$ \\
\hline $\mathrm{F} 8 \mathrm{BT}^{+}$ & 725 & $49,000 \pm 5,000$ & CMS (film) \\
\hline $\mathrm{pF}^{-\bullet}$ & 600 & $62,000 \pm 10,000$ & PR in benzene with benzophenone ${ }^{a)}$ \\
\hline $\mathrm{pF}^{-\bullet}$ & 597 & $69,000 \pm 7,000$ & Chemical reduction in THF with $\mathrm{NaBP}$ ) \\
\hline
\end{tabular}




$\begin{array}{cccc}\mathrm{pF}^{*} & 600 & 70,000 \pm 15,000 & \text { PR in THF }^{\mathrm{b})} \\ \mathrm{pF}^{*} & 599 & 86,000 \pm 10,000 & \mathrm{CMS}(\text { film }) \\ \mathrm{pF}^{+\cdot} & 580 & 60,000 \pm 10,000 & \text { PR in benzene using TTA } \\ \mathrm{pF}^{+\cdot} & 577 & 62,000 \pm 10,000 & \text { PR in DCE } \\ \mathrm{pF}^{+\cdot} & 626 & 90,000 \pm 2,000 & \text { CMS (film) }\end{array}$

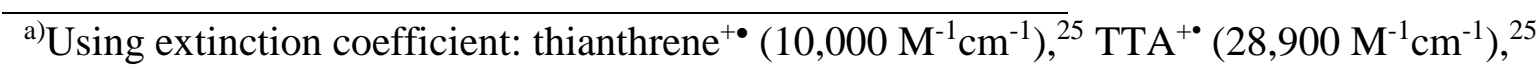
benzophenone ${ }^{-\bullet}\left(8,600 \mathrm{M}^{-1} \mathrm{~cm}^{-1}\right),{ }^{26}$ biphenyl $^{-\bullet}\left(12,500 \mathrm{M}^{-1} \mathrm{~cm}^{-1}\right),{ }^{27}$ b) ${ }^{2}$ rom reference ${ }^{14}$

Spectra obtained by pulse radiolysis in benzene were taken at 1 - $5 \mu$ s after the electron pulse, in solutions containing 0.5 - $2 \mathrm{mM}$ (PRU) polymer with 0.5 - $1 \mathrm{mM}$ of either tetrafluorobenzoquinone (F4BQ) or Ferrocene (Fc) for the hole and electron spectra respectively. The redox potentials let F4BQ remove electrons but not holes and let Fc remove holes but not electrons. Both F4BQ and Fc also remove the polymer triplet excited state which is formed during pulse radiolysis in benzene (see Table 2 for redox potentials and triplet energies and Experimental Section for further details). F4BQ anions have an absorption peak at $450 \mathrm{~nm}(2.76 \mathrm{eV})$ with a tail that may extend above $500 \mathrm{~nm}$ (below $2.48 \mathrm{eV}$ ) which may contribute small errors to the polymer cation spectra in those regions. Ferrocene cations have an absorption at $617 \mathrm{~nm}$ but with a much smaller extinction coefficient of $410 \mathrm{M}^{-1} \mathrm{~cm}^{-1}$ so would contribute errors to the polymer anion spectra near $617 \mathrm{~nm}$, but these will be negligible.

Table 2. Reduction potentials and triplet energies

\begin{tabular}{|c|c|c|c|}
\hline $\begin{array}{l}\text { Molecule I } \\
\text { Polymer }\end{array}$ & $\begin{array}{c}\text { Redox Potential } \\
\text { vs Fc/Fc }{ }^{+}(0 /-) \\
{[\mathrm{V}]}\end{array}$ & $\begin{array}{c}\text { Redox Potential } \\
\text { vs Fc/Fc }{ }^{+}(+/ 0) \\
{[\mathrm{V}]}\end{array}$ & $\begin{array}{c}\text { Triplet } \\
\text { Energy } \\
{[\mathrm{eV}]}\end{array}$ \\
\hline $\mathrm{pF}$ & -2.65 & 0.66 & 2.24 \\
\hline $\mathrm{F} 8 \mathrm{~B} \mathrm{~T}^{\star}$ & $-1.9 /-2$ & 0.8 & $\geq 1.6$ \\
\hline Fc & & 0 & 1.65 \\
\hline F4BQ & -0.45 & & 2.23 \\
\hline
\end{tabular}

$\mathrm{pF}$ redox values are taken from one electron charge transfer equilibrium measurements with molecules of known redox potentials. ${ }^{14}$ F8BT redox values are from the widely used HOMO value of $5.9 \mathrm{eV}$, originating from R. B. Fletcher PhD Thesis (Sheffield, 1999) cited first in reference, ${ }^{28}$ converted back to redox potential vs $\mathrm{Fc} / \mathrm{Fc}+$ using constant of $5.1 \mathrm{eV}$ as suggested from reference. ${ }^{29} *$ F8BT values have significant uncertainty. F8BT LUMO has been 
estimated to be $3.1 \mathrm{eV}^{30}$ and $3.2 \mathrm{eV},{ }^{28}$ based on the optical band gap and estimate of exciton binding energy. An independent measurement of cyclic voltammetry of F8BT (deposited on electrode) in $0.1 \mathrm{M}$ solution of tetrabutylammonium hexafluorophosphate in acetonitrile vs

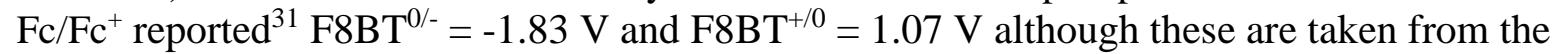
peak currents rather than onsets as is widely used. A further source (Book: Organic Light Emitting Devices: Synthesis, Properties and Applications) cites PhD thesis of Arne Morteani (Cambridge, 2004) to give HOMO/LUMO levels of $5.74 \mathrm{eV}$ and $2.94 \mathrm{eV}$ respectively. F4BQ redox value based on $\mathrm{F} \mathrm{BQ} \mathrm{Q}^{0 /-}=-0.04 \mathrm{~V}$ vs SCE and $\mathrm{Fc}^{+/ 0}=0.41 \mathrm{~V}$ vs SCE in MeCN. ${ }^{32} \mathrm{pF}$ triplet energy is the average from references. ${ }^{33-34}$

F8BT triplet energy is a lower limit based on the fact that, F8BT triplet reacted with Fc with a rate constant of $2.5 \times 10^{9} \mathrm{M}^{-1} \mathrm{~s}^{-1}$ suggesting it is either in equilibrium with $\mathrm{Fc}$, which has a reported triplet lifetime of $0.6 \mathrm{~ns},{ }^{35}$ or the Fc triplet energy is lower. Ferrocene triplet reference. ${ }^{36}$ F4BQ triplet reference ${ }^{37}$ (via reference ${ }^{38}$ ).

Anion and cation spectra were also measured using pulse radiolysis in more polar solvents, THF $\left(\varepsilon_{\mathrm{r}}=7.58\right)$ and ODCB $\left(\varepsilon_{\mathrm{r}}=9.93\right)$ respectively, without the need for scavengers due to the solvent radiation chemistry which produces only electrons in THF and only holes in ODCB. Many of the polymer cations formed in ODCB are likely to be paired with chloride ions as the $\left(\mathrm{P}^{\bullet+}, \mathrm{Cl}^{-}\right)$ion pair is favored energetically over recombination.

Chemically reduced and oxidized spectra from the earliest stages of the titration are shown in Figure 4 with the counter-ion noted in parenthesis.

\section{Microwave Conductivity}




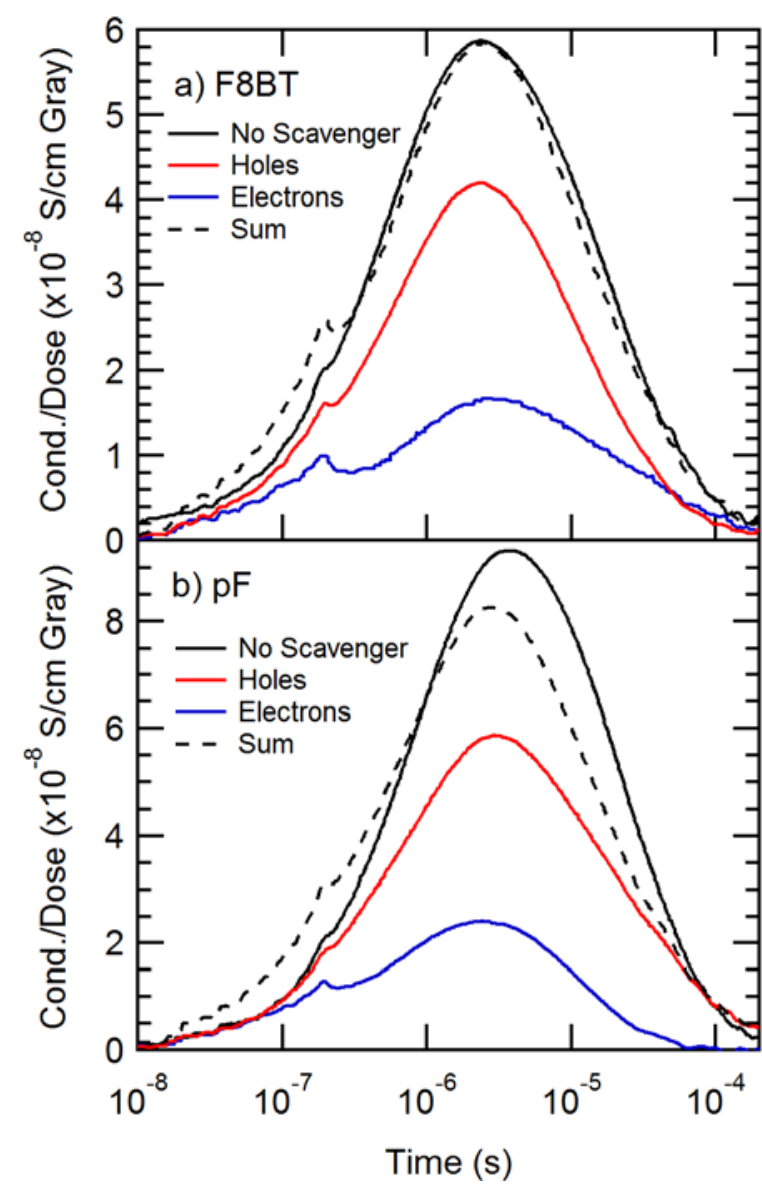

Figure 5. Microwave conductivity $(8.7 \mathrm{GHz})$ per unit dose from argon-purged benzene solutions containing $0.5 \mathrm{mM}$ (PRU) of a) F8BT and b) pF. All solutions contain $5 \mathrm{mM}$ biphenyl. Black traces are when no scavenger is present. For isolating hole and electron conductivity, $0.5 \mathrm{mM}$ of F4BQ or Ferrocene were added respectively. The dashed line is the sum of conductivity from the hole and electron traces. Ionizations were formed by a $2 \mathrm{MeV}$ electron pulse of 200 ns duration. Note, these conductivity traces alone do not reflect the relative mobility values as the charge concentrations were found to be different for electrons and holes from transient absorption measurements (see Supporting Information).

Figure 5 shows transient microwave conductivity traces for $0.5 \mathrm{mM}(\mathrm{PRU}) \mathrm{F} 8 \mathrm{BT}$ and $\mathrm{pF}$ solutions in dry, oxygen-free benzene following a $200 \mathrm{~ns} 2 \mathrm{MeV}$ electron pulse with either no scavenger, an electron scavenger (F4BQ, $0.5 \mathrm{mM}$ ), or a hole scavenger (Ferrocene, $0.5 \mathrm{mM}$ ). At any given microwave frequency both real and imaginary components of conductivity can contribute to changes in the TRMC signal due to either absorption of microwaves or a shift in the resonant frequency of the cavity respectively. The conductivity shown in Figure 5 is the real part of the complex conductivity that was extracted from a global fit to 30 - 40 transients over the range 8.65 - $8.9 \mathrm{GHz}$. All solutions also contained $5 \mathrm{mM}$ of biphenyl as a mediator 
for electrons between the solvent and the polymers as the yield of solvent electrons captured is strongly dependent on attachment rates to the solute due to competing reactions; the biphenyl is intended to capture a consistent yield of electrons in each solution, preventing a lot of the fast non-geminate ion-recombination by rapidly-moving quasi-free electrons in benzene $\left(\mu_{\mathrm{e}}=0.14 \mathrm{~cm}^{2} / \mathrm{Vs}\right) .{ }^{39}$ The biphenyl anions live long enough to reduce other solutes, such as the polymers, at lower concentrations. Figure 5a confirms the consistent yield of ions, as the sum of the F8BT electron and hole conductivity is equal to the trace with no scavenger. To determine the absolute concentration of charges, $N$, transient absorption ${ }^{40}$ measurements were performed on the same solutions (Supporting Information) resulting in a calculated mobility ratio of $\mu_{\mathrm{h}}=2.7 \mu_{\mathrm{e}}$. The absolute values of intramolecular mobility are estimated to be $\mu_{1 \mathrm{D}}=0.09 \mathrm{~cm}^{2} / \mathrm{Vs}$ and $\mu_{1 \mathrm{D}}=0.24 \mathrm{~cm}^{2} / \mathrm{Vs}$ for electrons and holes respectively in F8BT, using $\mu_{1 \mathrm{D}}=3 \sigma / \mathrm{Ne}$ where $e$ is the charge on an electron. These values represent a lower limit as chain curvature and finite chain length can lower the measured mobility with respect to the true intrinsic mobility. Detailed measurements of hole mobility on polyfluorene chains suggest the intrinsic mobility may be three times larger than the values of $\mu_{1 \mathrm{D}}$ measured on the longest chains, ${ }^{40}$ although the ratio of $\mu_{\mathrm{h}}: \mu_{\mathrm{e}}$ is expected to be weakly dependent on chain length if they are similar.

The conductivity traces for $\mathrm{pF}$ in Figure $5 \mathrm{~b}$ show that the $\mathrm{pF}$ anions decay more rapidly than any of the other polymer ions. Electrons in the pF transistors for CMS also became trapped more rapidly than any of the other species. The common reason is most likely due to the large, negative reduction potential of $\mathrm{pF}$, or equivalently a high lying LUMO level, which exposes it to a larger range of possible impurities that could take the excess electron from it. Electron transfer to oxygen is a possible cause for the loss of $\mathrm{pF}$ anion although F8BT anions should also react with $\mathrm{O}_{2}$, so it is logical to look to other causes. A fluorenone defect on some of the chains, an unknown impurity in the solvent, or an impurity in the polyfluorene material may be to blame. For $\mathrm{pF}$ the sum of the separate electron and hole conductivity 
traces is smaller than the total in the 'no scavenger' trace. This may be due to different concentrations of some electron-scavenging impurity in the two solutions. To check the concentration of polymer ions in each solution, PR-TAS was performed following the microwave conductivity measurement on the same solutions (see Supporting Information). Optical absorption from the polarons indicated that the concentration of electrons was indeed lower than that of holes however, not by enough to suggest that the mobilities of electron and hole are the same. For polyfluorene we find a mobility ratio of $\mu_{\mathrm{h}}=(1.5 \pm 0.2) \mu_{\mathrm{e}}$. The absolute values of mobilities for this particular batch of polyfluorene were $\mu_{1 \mathrm{D}}=0.2 \mathrm{~cm}^{2} / \mathrm{Vs}$ and $\mu_{1 \mathrm{D}}=0.3 \mathrm{~cm}^{2} / \mathrm{Vs}$ for electrons and holes respectively. Fluorenone defects, if present, would be expected to trap electrons in polyfluorene: Computations $(\omega \mathrm{PBE}, \omega=0.1,6-31 \mathrm{G} *$ in benzene) for an $F_{5}$ oligomer with (or without) the central unit converted to fluorenone predict a $0.7 \mathrm{eV}$ trapping energy. But fluorenone defects would not capture holes based on fluorenone’s IP $(8.3 \mathrm{eV}),{ }^{41}$ which is higher than that for fluorene monomer. ${ }^{42}$ If just $1 \%$ of repeat units had such a defect, and the average chain length is 30 PRU, 26\% of chains could have at least one electron trap, so fluorenone defects could readily account for the lower observed concentration of polymer anions although not necessarily the lower electron mobility as the trap depth is many times the thermal energy at room temperature and so would be considered a deep trap.

\section{DISCUSSION}

Electron Localization vs. Hole Delocalization. Table 3 reports delocalization lengths $\left(l_{n}\right)$ and extinction coefficients of $\mathrm{F} \mathrm{BT}^{\circ-}$ polarons in THF and F8BT ${ }^{\circ+}$ in ODCB. The extinction coefficients are from pulse radiolysis experiments which add one electron or hole to a chain. Titration spectra and pulse radiolysis determine delocalization length as described previously by determining the number of F8BT repeat units of the neutral bleached upon addition of one electron or hole. ${ }^{2}$ 
Table 3. Delocalization lengths $\left(l_{\mathrm{n}}\right)$ of $\mathrm{F} \mathrm{BT}^{\circ-}$ in THF and F8BT ${ }^{*+}$ in ODCB and the molar extinction coefficients $(\varepsilon)$ at their maximum absorption, $\lambda_{\max }$, used to determine them. Values in the parenthesis for $\mathrm{FBBT}^{*}$ - were measured in the presence of $\mathrm{C}_{222}$.

\begin{tabular}{ccc}
\hline & $\begin{array}{c}\varepsilon \\
{\left[10^{4} \mathrm{M}^{-1} \mathrm{~cm}^{-1}\right]}\end{array}$ & $\begin{array}{c}I_{\mathrm{n}} \\
{[\mathrm{PRU}]}\end{array}$ \\
\hline $\mathrm{F} 8 \mathrm{BBT}^{-}(\mathrm{PR})$ & $3.04 \pm 0.60^{\mathrm{a})}(2.67 \pm 0.28)^{\mathrm{b})}$ & $1.36 \pm 0.37^{\mathrm{c})}(2.02 \pm 0.10)^{\mathrm{d})}$ \\
${\mathrm{F} 8 \mathrm{~B} T^{-} \text {(titration) }}$ & $2.60 \pm 0.26(2.25 \pm 0.10)$ & $1.16 \pm 0.32(1.70 \pm 0.08)$ \\
$\mathrm{F} 8 \mathrm{BBT}^{\circ+}$ (titration) & $4.73 \pm 1.92$ & $3.18 \pm 1.29$ \\
$\mathrm{~F} 8 \mathrm{BBT}^{++}$(PR) & $5.5 \pm 1.0^{\mathrm{a})}$ & $4.3 \pm 0.78$ \\
\hline
\end{tabular}

a) Extinction coefficient measured by pulse radiolysis in THF containing sodium tetraphenylborate; ${ }^{\text {b) }}$ Same as a) but with $\mathrm{C}_{222}$ added; ${ }^{\mathrm{c})}$ Delocalization length in PRU from titration data using $\varepsilon$ determined by pulse radiolysis; ${ }^{\text {d) }}$ Same as c) but with $\mathrm{C}_{222}$ added.

Delocalization lengths were estimated from the spectra as the number of neutral repeat units removed for each electron or hole added to the polymer, finding that an electron in F8BT occupies $1.36 \pm 0.51$ repeat units (PRU) in the absence of $\mathrm{C}_{222}$ and $2.02 \pm 0.21 \mathrm{PRU}$ in the presence of $\mathrm{C}_{222}$. Uncertainties can be large when absorption bands of the anion or cation overlap with that of the neutral; the estimated uncertainties include estimates from this source. The inset to Figure 3 finds that the growth of $\mathrm{F} \mathrm{BT}^{\circ+}$ is described reasonably well with the assumption that each $\mathrm{F}_{\mathrm{BBT}}{ }^{++}$occupies 4.3 PRU, the value determined from neutral depletion in the titration. This assumption removes F8BT neutral faster in the equilibrium expression. The model calculation did not attempt to take into account Coulomb repulsion between the $\mathrm{F} \mathrm{BT}^{*+}$ polarons or the entropy of arranging the polarons in the chain, but the model does support the delocalization length. These results are consistent with the calculation shown in Figure 6, which shows the singly-occupied Kohn-Sham (KS) molecular orbital of the anion to be confined to one BT unit with slight density on the two adjacent fluorenes. A similar computation in Figure S7 finds the hole in $\mathrm{F}_{\mathrm{BBT}}{ }^{\circ+}$ to be more delocalized. 

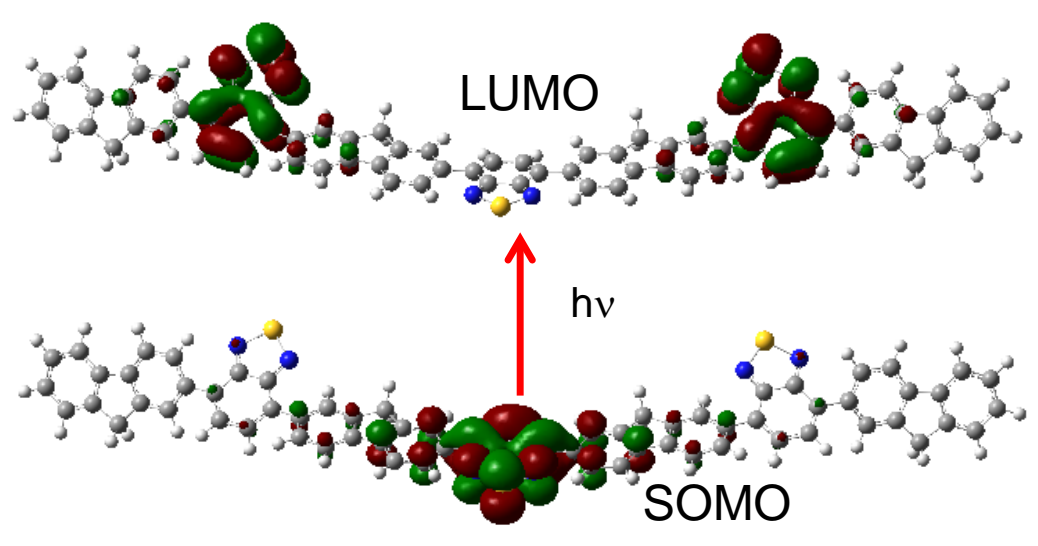

Figure 6. Isodensity plots of the principal KS orbitals contributing to the low energy $\left(\mathrm{P}_{1}\right)$ transition in the anion of F4BT3 oligomer in THF, serving as a model for anions of F8BT. The computed transition at $0.60 \mathrm{eV}$ has an oscillator strength $\mathrm{f}=0.26$ and is composed principally of a transition from the SOMO, on one BT group to the LUMO, on adjacent BT groups, and to the LUMO +1, which is similar Higher energy transitions, associated with the visible band, move electrons from the $\mathrm{BT}^{-}$group to orbitals having density on both fluorene (F) and BT groups; they are similar to those illustrated in Figure S9.

The possibility of a $\mathrm{P}_{3}$ band, ${ }^{43-45}$ absorbing at an energy close to the transition of the neutral (see Figure 3 and Figure S5), complicates the estimation of $l_{n}$ for an F8BT ${ }^{\bullet+}$ polaron. We can place bounds on $l_{n}$ for $\mathrm{F}_{8 \mathrm{BT}}{ }^{\bullet+}$ using two extreme assumptions about the possible $\mathrm{P}_{3}$ band. If we assume that there is no $\mathrm{P}_{3}$ band so the transition at $2.7 \mathrm{eV}$ in Figure 3 is solely due to remaining F8BT neutral, then from an average of the values in Table $3, l_{n}=3.7 \mathrm{RU}$ (4.8 $\mathrm{nm})$. An alternative assumption can also be consistent with the data, in which the neutral band shifted blue and never decreased below $50 \%$ of its initial intensity in Figure 3. The data could be consistent with the possibility that a $\mathrm{P}_{3}$ exists and has an extinction coefficient half as large as the neutral. This assumption is further supported by chemical oxidation by $\mathrm{NO}^{+} \mathrm{PF}_{6}{ }^{-}$in Figure S5, which supports the existence of the $\mathrm{P}_{3}$. With this assumption $l_{n} \leq 7.4$ RU (9.6 nm).

Electronic Couplings and Barriers. We interpreted the low energy band of F8BT ${ }^{\circ-}$ in THF

(Figure 2) as a charge transfer (CT) band taking an electron localized on a BT group to an adjacent, neutral, BT group. Such a CT band is possible due to electronic coupling transmitted

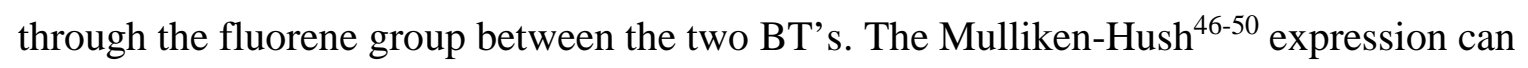


give an approximate estimate of this electronic coupling between BT groups, $\mathrm{H}_{\mathrm{da}}$, from spectroscopic parameters:

$H_{d a}=2.06 \times 10^{-2} \frac{\sqrt{v_{\max } \varepsilon_{\max } \Delta v_{1 / 2}^{0}}}{\mathrm{r}_{d a}}$

In Equation $1 v_{\max }=0.83 \mathrm{eV}$ is the energy at the maximum (peak) of the CT band, $\varepsilon_{\max }$ $=3807 \mathrm{M}^{-1} \mathrm{~cm}^{-1}$ is the molar extinction coefficient at the maximum, $\Delta v_{1 / 2}=0.66 \mathrm{eV}$ is the full width at half max for the CT band and $r_{d a}=13 \AA$ is the distance between two BT units in Angstroms. The constant applies for energies in $\mathrm{cm}^{-1}$ or eV. Application of Equation 1, to the near IR band of F8BT $^{*-}$ yields $\mathrm{H}_{\mathrm{da}}(\mathrm{BT}-\mathrm{BT})=72 \mathrm{meV} \pm 30 \%$. Experimental measurement of the weak band yields the substantial uncertainty. The reorganization energy, $\lambda$, is expected, in a two-state model, to equal $0.83 \mathrm{eV}$, the energy at the maximum of the band, but $\lambda$ may also be obtained from the band width, although the two estimates will give the same value when $\lambda$ arises entirely from classical modes:

$\Delta v_{1 / 2}=2 \sqrt{4 \ln (2) \lambda R T}$

Equation 2 gives a larger value $\lambda=1.5 \mathrm{eV}$. This discrepancy tells us that the width of the band contains contributions from sources other than the classical fluctuations included in Equation 2. Additional contributions could come from inhomogeneities arising from varying chain conformations, from reorganization in high frequency skeletal vibrational modes or from the need to use a three state model to include effects of the fluorene bridge between the two BT groups. We assume that these effects do not introduce errors into the estimates of electronic coupling from Equation 1 larger than those from experimental uncertainties, and, that while vexing, this discrepancy does not call into question the interpretation of the band as a CT transition. The electronic coupling, $\mathrm{H}_{\mathrm{da}}(\mathrm{BT}-\mathrm{BT})=72 \mathrm{meV} \pm 30 \%$, can alternatively be estimated by computation on simplified model structures.

Figure 7 shows computed energy levels for electron and hole attachment to isolated BT and F groups, and couplings computed from splittings in symmetric F-BT-F and BT-F-BT 
molecules. This computation finds that the LUMO (on F) lies $1.7 \mathrm{eV}$ above the LUMO (on $\mathrm{BT}$ ) while the equivalent gap between the HOMOs is $0.61 \mathrm{eV}$. This calculation thus finds a larger barrier to electron transfer between two BT's than for hole transfer between two F's, compatible with the report by Cornil et. al. ${ }^{18}$ The computed value, $\mathrm{H}_{\mathrm{da}}(\mathrm{BT}-\mathrm{BT})=54 \mathrm{meV}$ is slightly less than the Mulliken-Hush estimate from the CT band. The much larger $\mathrm{H}_{\mathrm{da}}(\mathrm{F}-\mathrm{F})=$ $259 \mathrm{meV}$ can be understood in terms of the smaller energy barrier and the smaller distance between adjacent F units. Therefore, an electron is more likely to be localized into one BT unit. The larger $\mathrm{H}_{\mathrm{da}}(\mathrm{F}-\mathrm{F})$ for the HOMOs can more readily delocalize a hole polaron, in agreement with the finding that holes are moderately delocalized, even in polar oDCB.


Figure 7. Electronic couplings in F8BT estimated from models BTFBT and FBTF. Couplings between two BT groups separated by an F are $54 \mathrm{meV}$, but a much larger coupling, $259 \mathrm{meV}$, is found between two F's separated by a BT. These are Koopmans' theorem couplings estimated in b3lyp/6-31g(d) calculations on neutral molecules from splittings of the LUMOs in the BT case and HOMOs in the F case. The couplings between F's might be expected to be larger because a bridging BT separates the two Fs by a short distance and presents a smaller barrier $(0.61 \mathrm{eV})$, while the $1.7 \mathrm{eV}$ energy difference between BT and F LUMOs presents a larger energy barrier.

Evolution of Absorption Bands with Extent, n, of Reduction or Oxidation. F8BT neutrals exhibit two electronic transitions at 2.7 and $3.9 \mathrm{eV}$ in Figure 2, and 3. Jespersen and 
coworkers $^{51}$ used ZINDO calculations on an F8BT oligomer; having 5 F and 4 BT units to conclude that the low energy band $(2.7 \mathrm{eV})$ is likely due to a charge transfer from an $\mathrm{F}$ to create an exciton localized on one BT unit. They proposed that the high energy band (3.9 eV) is due to a transition from the HOMO to the LUMO in which case the excitation is delocalized into the whole chain.

F8BT $^{\circ-}$ polarons in THF in the presence of $\mathrm{C}_{222}$ in Figure 2 possess a weak transition in the NIR region, identified here as a charge transfer band. The intensity of this band increases to a maximum near $n=0.5$ electrons per repeat unit followed by a decrease in intensity with further reduction. This behavior contrasts with that of the prominent visible band peaking near $2.0 \mathrm{eV}$ which increases almost linearly to a maximum near $\mathrm{n}=1.0$, as does another at $3.15 \mathrm{eV}$. Similarly the two prominent bands of F8BT neutral decrease to minima near $n=1.0$. These growths in the visible, peaking at $n=1.0$, accompanied by bleaches of F8BT neutral bands are easily understood as due to formation of F8BT $^{\circ-}$ polarons that occupy one repeat unit. To explain the exceptional behavior of the NIR band we first examine the nature of the transition. TDDFT calculations find the low energy transition to take the electron localized on one BT group from the SOMO and transfer it to LUMOs on neighboring BT groups (Figure 6). Like the experimental interpretation, the computations thus identify the NIR band as due to a charge transfer (CT) transition that principally moves an electron localized on one BT group to other BT groups. The computed oscillator strength, $f=0.26$, is small compared to $\mathrm{f}>1.0$ typical of delocalized polarons; the small oscillator strength is typical for a CT transition between two groups some distance apart. The polar THF solvent included in the calculation via a reaction field contributes to the localization. The degree of localization would likely have been even more complete if the $\mathrm{Na}^{+}$counter-ion were included in the calculation.

The CT nature of the NIR transition can explain its curious evolution with n. Because the transition transfers an electron to another BT group, at low doping, $\mathrm{n}<\sim 0.3$, each $\mathrm{F}^{\mathrm{B} T}{ }^{\circ-}$ 
polaron can transfer an electron to an empty BT either to its left or to its right, in agreement with the TDDFT result in Figure 6. When half of the BT groups are occupied by electrons (n $=0.5$ ), the probability that a neighboring BT is occupied by an electron, and therefore not available to receive an electron, is 0.5 . Before $n=0.5$ the absorbance increases with $n$, but at $n$ $=0.5$ it reaches a maximum and for $\mathrm{n}>0.5$ it decreases. The different behavior, peaking at $\mathrm{n}$ $=1$, identifies the visible bands as transitions within an $\mathrm{F} 8 \mathrm{BT}^{\circ-}$ polaron, not charge transfer transitions to neighboring groups. TDDFT calculations on an oligomer with all BT's reduced ( $\mathrm{n}=1.0)$ support this interpretation, finding a strong computed transition at $1.87 \mathrm{eV}$, which is near to the observed band at $2.0 \mathrm{eV}$ (see Figure S6).

In reductive doping, the evolution of the visible $\left(\mathrm{P}_{2}\right)$ band is very sensitive to ion pairing. Noticeable spectral shifts occur in the absence of $\mathrm{C}_{222}$ in Figure $\mathrm{S} 6$ as previously observed for small negative polarons like PPV $(2 \mathrm{~nm}) .^{2}$ The dissociation constant is likely to be small due to the small size of $\mathrm{FBBT}^{*-}$, which is near to the size of a biphenyl molecule. Slates and Szwarc ${ }^{52}$ found the dissociation constant, $\mathrm{K}_{\mathrm{d}}\left(\right.$ biphenyl $\left.^{--}, \mathrm{Na}^{+}\right)=1.5 \times 10^{-6} \mathrm{M}$. Taking this as an estimate for $\mathrm{K}_{\mathrm{d}}\left(\mathrm{F}_{\mathrm{BBT}}{ }^{\circ-}, \mathrm{Na}^{+}\right)$, almost every $\mathrm{F} \mathrm{BT}^{\circ-}$ would be ion paired above a concentration of a few $\mu \mathrm{M}$. This contrasts to the behavior of longer polarons such as pF's and LPPP which have polarons with average lengths of 4-6 nm. In similar titrations both $\mathrm{P}_{1}$ and $\mathrm{P}_{2}$ bands exhibited growths of with very little shift in their spectra suggesting the possibility that principally free polarons are formed.

In contrast to $\mathrm{F} 8 \mathrm{BT}^{*-}$ electron polarons, $\mathrm{F} \mathrm{BT}^{\circ+}$ polarons exhibit a strong $\mathrm{P}_{1} \mathrm{NIR}$ band as shown in Figure 3. This is a typical spectral feature observed for delocalized electron polarons such as in pF's and LPPP. In Figure 3 the higher energy neutral band at $2.7 \mathrm{eV}$ seems to increase again with a slight blue-shift after $n>0.55$ in Figure 3. This signals that an additional hole polaron band may occur in the region where the neutral absorbs. The oxidation by $\mathrm{NO}^{\cdot+} \mathrm{PF}_{6}{ }^{-}$in Figure $\mathrm{S} 5$ in Supporting Information supports this idea of the $\mathrm{P}_{3}$ band. In that figure, the increase of an additional band located at a slightly higher energy than the neutral is 
apparent with higher doping. The computation also shows that oscillator strengths of four transitions at $\sim 2.7 \mathrm{eV}$ add up to 0.57 hinting at a possibility of the additional band.

Polarons in NonPolar Media. Figure 8 displays computed spectra of F8BT ${ }^{\circ+}$ and F8BT $^{*-}$ in media having different polarities. Observed spectra of $\mathrm{pF}^{{ }^{+}}, \mathrm{pF}^{{ }^{-}-}$and $\mathrm{F} \mathrm{BT}^{\bullet+}$ (Figure 4) show a $\mathrm{P}_{2}$ visible absorption that is remarkably insensitive to the polarity of the environment and to whether or not there is a counter ion present. However, in nonpolar benzene these three ions show slightly weaker NIR bands compared to those in more polar THF or ODCB, but none reach a maximum within our observable wavelength range. Computed spectra in Figure 8 predict the presence of a large $\mathrm{F}_{\mathrm{BST}}{ }^{\circ+} \mathrm{NIR}$ band, computed to appear at energies below our detection limit supporting the possibility that substantial $\mathrm{P}_{1}$ bands are there, awaiting detection. The lower energy $\mathrm{P}_{1}$ band is associated with a more delocalized polaron. The F8BT anion spectrum is far more sensitive to polarity than the other three ions. As shown in Figure 4, the lower energy absorption for the F8BT anion, that we attribute to a CT absorption from a localized electron, appears to grow and red shift going from THF with counter ion, to THF with no counter ion, to benzene, evolving to look more like a delocalized polaron. The computed spectra in Figure 8 also indicate a similar trend and show that the electron on F8BT is highly susceptible to localizing forces, and may become fairly delocalized in a non-polar environment.

The different behavior of electrons and holes on F8BT illustrate the competition between two factors that can lower the energy of a charge on a polymer chain: delocalization, which lowers the electronic energy of the state, and localization which solvates the charge better. If the electronic coupling becomes too small, or the environment too polar, solvation wins and the charge becomes localized, as in the F8BT electron. The electronic coupling between the fluorene HOMO states, however, is sufficiently strong that delocalization wins for the F8BT hole in all polarities investigated here. 


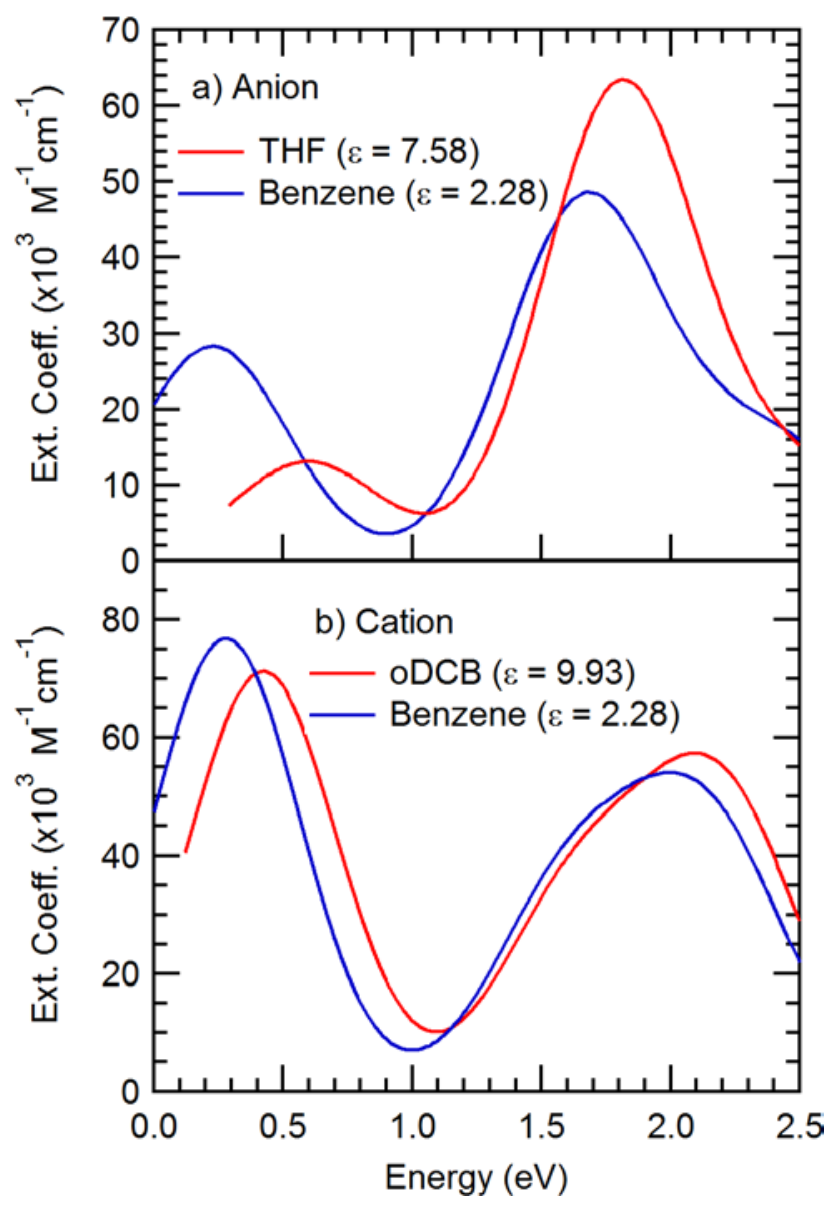

Figure 8. Predicted absorption spectra from DFT calculations of F8BT a) anions and b) cations, in solvents of different dielectric constant, using the range-corrected $\omega \mathrm{PBE}$ functional with $\omega=0.1 \mathrm{Bohr}^{-1}$ and the PCM solvation model for THF, benzene and ODCB. Gaussians with width, $w=0.4 \mathrm{eV}(\mathrm{FWHM}=2 \sqrt{\ln 2} w)$ and an area that satisfies the relationship between oscillator strength, $f$, and extinction coefficient, $\varepsilon\left(\mathrm{M}^{-1} \mathrm{~cm}^{-1}\right)$, as a function of wavenumber, $v\left(\mathrm{~cm}^{-1}\right)$, given by $f=4.319 \times 10^{-9} \int \varepsilon(v) d v$, were used to approximate the observed broadening in solution.

A striking feature of the F8BT CMS polaron spectra is the similarity to those measured in benzene, showing very little difference going from liquid to solid state, indicating that spectra obtained from pulse radiolysis in benzene, may be useful predictors of spectra in films. Exceptions would occur when there are significant morphological changes or electronic coupling to adjacent chains in the solid state, such as in regio-regular polythiophene. ${ }^{4-5,8}$ Indeed, Figure 4 shows that the $\mathrm{pF}$ cation spectrum in the solid state is quite different from that in benzene. A reasonable explanation would be the presence of beta phase $\mathrm{pF}$ in the films, which can be induced by exposure to n-butyl acetate, ${ }^{7}$ which was used to cast the PMMA 
dielectric layer from. The beta phase is likely to be the lowest energy site for charges to find due to planarization of the backbone caused by chain folding or aggregation and is responsible for changes in the absorption and emission spectra of neutral films. ${ }^{53-54}$ Only a partial CMS spectrum of the $\mathrm{pF}$ anion was obtainable due to the rapid trapping of electrons, even under vacuum, which caused the threshold voltage of the transistor to shift to more positive values, making it harder and harder to stay in accumulation and modulate the charge density. The pF anion CMS spectrum appears to be different from that of the corresponding cation spectrum, and closer to the spectrum found in benzene with pulse radiolysis, implying that anions may not occur in the beta phase. It is hard to conceive of why electrons do not appear to be going into the same beta phase regions as the holes do. One possible explanation would be that electrons do initially go into the beta phase segments, but get trapped by some mechanism leaving a fixed negative charge that prevents mobile electrons from entering that region, and being forced to stay in less ordered regions. Alternatively the absorption could be largely from electrons on fluorenone defects. Isolated fluorenone anions are known to absorb at $550 \mathrm{~nm},{ }^{26}$ and could end up absorbing somewhere close to polyfluorene when incorporated into a chain, however with an estimated trap depth of $0.7 \mathrm{eV}$, they are unlikely to be populated and depopulated at CMS frequencies. Finally, it could also be possible that beta phase pF constitutes a small fraction of the film in which holes are well stabilized but in which electrons are not substantially stabilized. It is well-known that radical cations of aromatic molecules are substantially stabilized by dimerization with neutrals $\mathrm{s}^{55-59}$ but dimers are not known for anions of the same molecules. It is thus conceivable that aggregation to form beta phase $\mathrm{pF}$ creates an environment that stabilizes holes more than it stabilizes electrons.

A further change that was observed when going from solution to films, in the case of polyfluorene, was in the magnitude of the extinction coefficient. An extinction coefficient would normally be representative of an average over all molecular orientations which would 
be the case in solution, but if the chain backbones lie preferentially in the plane of the films in the region where charges are, there could be an enhancement of up to $40 \%$ as the transition dipole is at 26.5 degrees to the polymer backbone. ${ }^{60}$ The polyfluorene films appears to show an enhancement with the extinction coefficient for $\mathrm{pF}$ cations going from $60,000 \mathrm{M}^{-1} \mathrm{~cm}^{-1}$ in benzene to $90,000 \mathrm{M}^{-1} \mathrm{~cm}^{-1}$ in the film. This result may be from of an enhancement in extinction coefficients for charges in beta phase $\mathrm{pF}$ due to a greater tendency for beta phase formation in the plane of the film at the interface with dielectric, or a combination of both. Determination of extinction coefficients herein thus provides a second type of evidence for hole formation in beta phase $\mathrm{pF}$. We also note that for the CMS experiment, the polyfluorene had octyl side chains, as opposed to the hexyl chains in all solution data reported here. Polyfluorene with octyl side chains shows a strong tendency to the planar beta phase formation whereas those with hexyl side chains do not. ${ }^{61}$ Within the accuracy of the measurements, no such change in extinction coefficient was observed for F8BT ions. This remarkable similarity suggests that F8BT ions are similar in solutions and films, and supports a random orientation in the film at the interface and no significant change in conformation in the solid state.

Transient Microwave Conductivity and Polarons. Transient microwave conductivity signals increase as mobilities increase. The observation that TRMC signals in benzene solution (Figure 5) for holes $\left(\mathrm{F} 8 \mathrm{BT}^{\circ+}\right)$ were nearly as large as that for hole in polyfluorene, a homopolymer, is consistent with the idea that holes are delocalized in F8BT and highly mobile. The true value for the intramolecular mobility is larger than the value measured from microwave conductivity, which is reduced by chain coiling, defects and short chain lengths. Estimating the true intramolecular mobility ideally requires a global fit to a series of different length chains, ${ }^{40}$ which would require length- resolved polymers and is thus beyond the scope of this paper. The ratio of hole and electron mobilities can however be determined accurately. The TRMC signal was smaller for electrons on F8BT, corresponding to a mobility of 0.09 
$\mathrm{cm}^{2} / \mathrm{Vs}$ which is close in magnitude to $0.08 \mathrm{~cm}^{2} / \mathrm{Vs}$, the mobility of electrons in liquid benzene at high pressure thought to occur by electron hopping between localized states. ${ }^{62-63}$ As charge transport is most likely limited by electronic coupling fluctuations through dihedral angles, it is not possible to say for sure that the electrons are localized or free. If a rigid, planar, push pull polymer could be measured, we could distinguish the effects of localization and corrugated potential more clearly. If holes and electrons were confined to single repeat units they would confront a corrugated potential, with barriers similar to those estimated in Figure 7. But if they delocalize over a few repeat units, the barriers would be greatly diminished. While we do not have measurements of delocalization lengths in this non-polar medium, the TRMC results imply that electrons in F8BT are substantially delocalized in benzene.

In Table 4. the mobility values from this work are displayed alongside a selection of literature values, made on films, of the same species. The differences between the single chain and film mobilities for these particular polymers illustrate the significant role that disorder in films has, and the difficulty in learning about the microscopic nature of charge carriers from bulk measurements.

Table 4. Summary of microwave mobility values from this work as compared to field effect transistor (FET) and Space Charge Limited Current (SCLC)

\begin{tabular}{|c|c|c|c|}
\hline \multirow{2}{*}{ Species } & \multicolumn{3}{|c|}{ Mobility $\left(\mathrm{cm}^{2} / \mathrm{Vs}\right)$} \\
\hline & PR-TRMC ${ }^{a}$ & FET & SCLC \\
\hline $\mathrm{pF}^{\bullet+}$ & 0.3 & $3 \times 10^{-4 b}$ & $10^{-7}-10^{-5 d}$ \\
\hline $\mathrm{pF}^{\bullet-}$ & 0.2 & $0.005-0.01^{b}$ & $10^{-6}-10^{-5} \mathrm{e}$ \\
\hline $\mathrm{F} 8 \mathrm{BT}^{\bullet+}$ & 0.24 & $10^{-4}-0.01^{c}$ & $3 \times 10^{-6 f}$ \\
\hline F8BT ${ }^{\circ-}$ & 0.09 & $10^{-4}-0.01^{c}$ & $3 \times 10^{-6 f}$ \\
\hline
\end{tabular}


a) This work. Lower limit of 1D 9 GHz mobility b) Ref. ${ }^{10,23}$ c) Refs, ${ }^{7,}{ }^{23}$ d) Refs. ${ }^{66-67}$ e) Ref. $^{64}$ f) Ref. ${ }^{21}$

\section{CONCLUSION}

Electrons in F8BT were localized on one BT unit in moderately polar environments. The absorption spectrum indicates a CT-type transition to neighboring BT unit and a polaron length just over one repeat unit. In contrast the delocalization length of the hole is closer to four repeat units and its optical spectrum has a more familiar polaron NIR $\mathrm{P}_{1}$ band.

The absorption spectrum of F8BT anion is very sensitive to solvent polarity and whether or not there is a counter ion. The trend of the NIR absorption suggests it becomes more delocalized as polarity is reduced. These results show how the localization of polarons in push-pull polymers is governed by a competition between two factors: electronic coupling (delocalizing) and solvent polarity (localizing). The F8BT anion is a good example where the electronic coupling is not sufficient to overcome the solvation energy gains from localization in a moderately polar environment.

Hole mobilities in $\mathrm{pF}$ and F8BT are remarkably similar in benzene solution despite the corrugated energy landscape in F8BT supporting the idea that charges in corrugated potentials may delocalize and move rapidly if the surrounding medium is nonpolar. The electron in F8BT is more localized than the hole and while spectroscopic evidence suggests the electron becomes more delocalized as the solvent polarity decreases, in benzene the electron mobility in F8BT is still lower than in $\mathrm{pF}$ by a factor of $\sim 3.3$. However, the absolute value of electron mobility in F8BT is still large $\left(\mu_{1 \mathrm{D}}=0.09 \mathrm{~cm}^{2} / \mathrm{Vs}\right)$ which supports the idea that in low polarity environments, charges will still delocalize sufficiently to enable good intra-chain mobility even in push-pull polymers with strong electron acceptors. Future work will seek to measure mobilities in other push-pull polymers after development of improved methods.

A longstanding question is the relation of polaron spectra in solution to those in films. This work finds nearly identical spectra in films and benzene solution for both F8BT $^{\circ+}$ and 
F8BT $^{*}$. The same is true for $\mathrm{pF}^{{ }^{*}}$, although the $\mathrm{P}_{2}$ band is narrower in films. For $\mathrm{pF}^{{ }^{+}}$, the film spectrum is sharper, shifted to lower energy and has a larger extinction coefficient. These changes may arise from beta phase formation in the $\mathrm{pF}$ films. Fluorenone or other defects may offer an explanation for why this is not observed for electrons in the pF films, but uncertainty remains. The current findings support the possibility that spectra of both electrons and holes in films are similar to their spectra in isolated chains in non-polar solutions except when specific morphological changes, such as beta phase formation for $\mathrm{pF}$ or aggregation in poly(hexylthiophene) are not present.

Findings described here for F8BT may apply to other push-pull (donor-acceptor) polymers.

\section{EXPERIMENTAL SECTION}

Chemical Doping: Poly[9,9-di-n-octylfluorenyl-2,7-diyl)-alt-(benzo[2,1,3]thiadiazol-4,8diyl)] (F8BT), the 2.2.2 cryptand 4,7,13,16,21,24-Hexaoxa-1,10-diazabicyclo [8.8.8] hexacosane $\left(\mathrm{C}_{222}\right)\left(\mathrm{Na}^{+} \mathrm{Bip}^{\bullet-}\right)$ were purchased from Aldrich; sodium biphenyl was from GF Smith. Chemical reductions were carried out in THF using a reducing agent, $\mathrm{Na}^{+} \mathrm{Bip}^{-}{ }^{-}$. In some cases the 2.2.2 cryptand, $\mathrm{C}_{222}$ was added to complex $\mathrm{Na}^{+}$ions. Chemical oxidations were carried out in ortho-dichlorobenze (ODCB). The oxidizing agent, thianthrenium hexafluorophosphate $\left(\mathrm{THI}^{\bullet+} \mathrm{PF}_{6}{ }^{-}\right)$, was prepared using a method similar to that of Wang Keun Lee. ${ }^{68}$ More details of sample preparation were described previously. ${ }^{2}$ After each addition of a reducing or oxidizing agent to $0.3 \mathrm{mM}$ polymer repeat units (PRU) of F8BT solution, UVVis-NIR absorption spectra were measured by Cary 5 spectrometer that scans from $200 \mathrm{~nm}$ to $3200 \mathrm{~nm}$. UV-Vis spectra were sometimes more conveniently recorded by an Ocean Optics fiber-coupled CCD spectrophotometer (200 to $900 \mathrm{~nm}$ ) inside the glove box.

Pulse Radiolysis Time Resolved Microwave Conductivity (PR-TRMC): PR-TRMC is an electrodeless method for determining the mobility, at GHz frequencies, of ions that have been created by ionizations from high energy electrons using pulse radiolysis. ${ }^{69-71}$ The method used here follows that described previously ${ }^{40}$ with the exception that a Hittite T2240 signal 
generator was used as a microwave source, allowing microwave absorption transients to be collected at precisely controlled frequencies covering the full extent of the resonant frequency dip of reflected power from the cavity that contains the solution. This new method of data collection, coupled with a fitting procedure that uses transmission line theory to simulate the reflected power, enables a global fit to be applied to all the microwave transients to recover the time dependent changes to the real and imaginary parts of the dielectric constant of the medium inside the cavity. For more information about the PR-TRMC technique, see Supporting Information.

Following pulse radiolysis of liquid benzene, electrons and holes are made in the solvent with mobilities of $0.14 \mathrm{~cm}^{2} / \mathrm{Vs}$ and $10^{-3} \mathrm{~cm}^{2} /$ Vs respectively. ${ }^{63,72]}$ Due to competing reactions of the ions in benzene, $5 \mathrm{mM}$ biphenyl (BP) was added to scavenge both electrons and holes quickly (reaction A) and then pass them on to the polymer (P) (reaction B) so that the yield of charges on the polymer would be nearly independent of the polymer concentration. The polymer solutions were $0.5 \mathrm{mM}$ in repeat unit concentration. To isolate the electron and hole mobility for the polymer, ferrocene (Fc) and tetrafluorobenzoquinone (F4BQ) were added at $0.5 \mathrm{mM}$ concentrations to scavenge holes and electrons respectively (reaction D and $\mathrm{E}$ ); the ions of these scavengers give negligible conductivity compared to the polymer ions. A further advantage of using ferrocene and F4BQ is that they also remove the polymer triplet states that were formed in reaction C. Triplets are transferred to ferrocene by triplet energy transfer (reaction F) whereas for F4BQ, triplets are removed from the polymer in a charge transfer process (reaction $G$ ). Reaction $G$ proposes that F4BQ quenches polymer triplets through formation of a charge transfer state, ${ }^{3}\left(\mathrm{P}^{\delta+}, \mathrm{F} 4 \mathrm{BQ}{ }^{\delta-}\right)$, similar to that reported for end-capped polymers. ${ }^{73}$ The redox potentials and triplet energies of the polymers and scavengers used in this study are shown in Table 2.

$$
\begin{aligned}
& \mathrm{e}^{-\bullet}+\mathrm{BP} \rightarrow \mathrm{BP}^{-\bullet} \\
& \mathrm{BP}^{-\bullet}+\mathrm{P} \rightarrow \mathrm{BP}+\mathrm{P}^{-\bullet}
\end{aligned}
$$


$\mathrm{P}^{-\bullet}+\mathrm{P}^{+\bullet} \rightarrow{ }^{1} \mathrm{P}^{*},{ }^{3} \mathrm{P}^{*}$

$\mathrm{P}^{+\bullet}+\mathrm{FC} \rightarrow \mathrm{P}+\mathrm{FC}^{+\bullet}$

$\mathrm{P}^{-\bullet}+\mathrm{F} 4 \mathrm{BQ} \rightarrow \mathrm{P}+\mathrm{F}^{-B} \mathrm{BQ}^{\bullet}$

${ }^{3} \mathrm{P}^{*}+\mathrm{FC} \rightarrow \mathrm{P}+{ }^{3} \mathrm{FC}^{*}$

${ }^{3} \mathrm{P}^{*}+\mathrm{F} 4 \mathrm{BQ} \rightarrow{ }^{3}\left(\mathrm{P}^{\delta+}, \mathrm{F} 4 \mathrm{BQ}{ }^{\delta-}\right) \rightarrow \mathrm{P}+\mathrm{F} 4 \mathrm{BQ}$

Note: This is not an exhaustive list of all possible reactions, but includes the most important ones.

Pulse Radiolysis Transient Absorption Spectroscopy (PR-TAS): Pulse radiolysis was performed with the Laser Electron Accelerator Facility (LEAF) at Brookhaven National Laboratory, ${ }^{74}$ which makes $10 \mathrm{MeV}$ electron pulses with $<50$ ps duration.

Transient absorption in the visible/near IR region was performed with light from a pulsed Xenon arc lamp that was focused into the solution counter-collinearly to the electron beam. Transmitted light was then passed through 10nm-width interference filters before being measured by various detectors depending on the wavelength: EG\&G FND-100Q ( $\mathrm{Si}, \lambda<1000$ nm), GPD Optoelectronics GAP-500 L (InGaAs, $1000 \mathrm{~nm}>\lambda>1700 \mathrm{~nm}$ ) or Sensors Unlimited SU500 (InGaAs, $\lambda>1700 \mathrm{~nm}$ ) photodiodes. The detector output was recorded with a Tektronix TDS-640Zi transient digitizer.

Charge Modulation Spectroscopy: The experimental setup for charge modulation spectroscopy is shown in Figure 1. Light from a Xenon lamp was passed through a monochromator and focused onto the OFET device, with the transmitted light being focused again onto a silicon detector. For wavelengths where the polymer absorbs, a long pass filter was used to stop fluorescence from the conjugated polymer from being picked up by the detector. A DC bias with a small AC modulation, typically of $37 \mathrm{~Hz}$, was applied to the OFET gate contact using a Keithley 3390 Waveform/Function Generator to modulate the charge density in the transistor while the device is held in accumulation. Two lock-in amplifiers 
(Stanford Research Systems SR 830) were used to detect changes in absorption and the current flowing on and off the device via the, shorted, source and drain electrodes. As well as taking a spectrum of the polarons while measuring the AC current, capacitance-voltage (CV) and capacitance-frequency scans (CF) could be performed with the charges being detected simultaneously both optically, at a fixed wavelength, and electrically in order to get accurate polaron absorption cross-sections. A patterned gate electrode is required for the accurate determination of charge density in the accumulation layer, particularly if the device exhibits large contact resistance.

In order to reduce electric field induced changes in transmission due to electrostriction, a Poly(3,4-ethylenedioxythiophene)-poly(styrenesulfonate) (PEDOT:PSS) gate electrode was used which had lower reflectivity than a metal gate electrode. To isolate and remove absorptions from modulations of the charge density in the PEDOT layer, OFETs with aligned semiconducting polymer layers were made (see Supporting Information for more detail) in the following way: polyimide (PI2525, HD Microsystems) was spin-coated onto $0.7 \mathrm{~mm}$ thick Corning $1737 \mathrm{~F}$ glass substrates and annealed for 3 hours at $300{ }^{\circ} \mathrm{C}$. Metal source and drain electrodes were then deposited by thermal evaporation through shadow masks in two steps giving a $40 \mu \mathrm{m}$ channel with a channel length of $13.4 \mathrm{~mm}$. To reduce optical transmission in the electrode region, where electroabsorption and electrostriction effects are strongest, but keep them as thin as possible $20 \mathrm{~nm}$ aluminum followed by $20 \mathrm{~nm}$ gold was used for the electrodes. To make the alignment layer, the polyimide-coated substrate was then rubbed with a cloth in an RM-50 rubbing machine (E.H.C. Co. Ltd) where the cylinder wrapped in cloth is rotating at $1200 \mathrm{rpm}$ as the substrate passes once under at $2.2 \mathrm{~mm} / \mathrm{s}$ before being placed in a nitrogen glovebox for film deposition. A $60 \mathrm{~nm}$ film of semiconducting polymer was spin coated under nitrogen from anhydrous xylene and annealed at above the melting temperature $\left(270-290{ }^{\circ} \mathrm{C}\right)$ for 20 minutes to align the polymer before being quenched on a metal surface at room temperature. A $~ 750 \mathrm{~nm}$ film of poly(methyl methacrylate) (PMMA) 
was spin coated from anhydrous n-butyl acetate and annealed at $80{ }^{\circ} \mathrm{C}$ for around 20 minutes to help to remove residual solvent. Patterned PEDOT:PSS gate electrodes were prepared by pre-evaporating less than $1 \mathrm{~nm}$ of aluminum onto the PMMA surface through a shadow mask and then spin-coating the PEDOT:PSS in air before annealing briefly in air at $100{ }^{\circ} \mathrm{C}$ and then returning to the glovebox for a further anneal at $120{ }^{\circ} \mathrm{C}$ for 1 hour. Due to changes in surface wettability, the PEDOT:PSS only remained on the aluminum-patterned area which formed a rectangle just above the source and drain electrodes with a thin connector to a contact pad.

\section{ASSOCIATED CONTENT}

\section{Supporting Information}

The Supporting Information is available free of charge on the ACS Publications website at DOI:

Obtaining real part of microwave conductivity, technique to minimize electrostriction effects in CMS measurements, determination of charge concentrations for accurate mobility values, oxidation of F8BT with $\mathrm{NOPF}_{6}$ in $\mathrm{oDCB}$, reduction of F8BT in THF with $\mathrm{Na}^{+} \mathrm{Bip}^{\bullet-}$ with and without $\mathrm{C}_{222}$, isodensity plot of F8BT cation, and schematic explanation of change in F8BT anion CT absorption as a function of degree of reduction of the chain. (PDF)

\section{AUTHOR INFORMATION \\ Corresponding Author}

*E-mail: mbird@bnl.gov

\section{ACKNOWLEDGMENTS}

This work, and use of LEAF and the Van de Graaff facility of the BNL Accelerator Center for Energy Research, was supported by the US Department of Energy (DOE), Office of Science, Office of Basic Energy Sciences, Division of Chemical Sciences, Geosciences \& Biosciences (CSGB) under Contract No. DE-AC02-98-CH10886. Work to develop the Pulse-Radiolysis TRMC experiment was supported by Laboratory Directed Research Grants 02544 at BNL and 06RF1002 at NREL in collaboration with Obadiah Reid and Garry Rumbles. M.B. and H.S. 
acknowledge support from EPSRC grant number EP/G060738/1 for the charge modulation spectroscopy experiments.

M.B. would like to thank Professor Ni Zhao and Professor Jana Zaumseil for introduction to the CMS technique.

\section{REFERENCES}

1. Duan, C.; Huang, F.; Cao, Y., Recent Development of Push-Pull Conjugated Polymers for Bulk-Heterojunction Photovoltaics: Rational Design and Fine Tailoring of Molecular Structures. J. Mater. Chem. 2012, 22, 10416-10434.

2. Bakalis, J.; Cook, A. R.; Asaoka, S.; Forster, M.; Scherf, U.; Miller, J. R., Polarons, Compressed Polarons, and Bipolarons in Conjugated Polymers. J. Phys. Chem. C 2014, 118, 114-125.

3. Fesser, K.; Bishop, A. R.; Campbell, D. K., Optical-Absorption from Polarons in a Model of Polyacetylene. Phys. Rev. B 1983, 27, 4804-4825.

4. $\quad$ Sirringhaus, H.; Brown, P. J.; Friend, R. H.; Nielsen, M. M.; Bechgaard, K.; Langeveld-Voss, B. M. W.; Spiering, A. J. H.; Janssen, R. A. J.; Meijer, E. W.; Herwig, P., et al., Two-Dimensional Charge Transport in Self-Organized, High-Mobility Conjugated Polymers. Nature 1999, 401, 685-688.

5. Brown, P. J.; Sirringhaus, H.; Harrison, M.; Shkunov, M.; Friend, R. H., Optical Spectroscopy of Field-Induced Charge in Self-Organized High Mobility Poly(3Hexylthiophene). Phys. Rev. B 2001, 63, 125204.

6. Deng, Y. Y.; Sirringhaus, H., Optical Absorptions of Polyfluorene Transistors. Phys. Rev. B 2005, 72, 045207.

7. Bird, M. Optoelectronic Processes in Polyfluorene Ambipolar 
Transistors. University of Cambridge, Cambridge, UK, 2010.

8. $\quad$ Beljonne, D.; Cornil, J.; Sirringhaus, H.; Brown, P. J.; Shkunov, M.; Friend, R. H.;

Bredas, J. L., Optical Signature of Delocalized Polarons in Conjugated Polymers. Adv. Funct. Mater. 2001, 11, 229-234.

9. Chin, X. Y.; Yin, J.; Wang, Z.; Caironi, M.; Soci, C., Mapping Polarons in Polymer Fets by Charge Modulation Microscopy in the Mid-Infrared. Sci. Rep. 2014, 4, 3626.

10. Chua, L.-L.; Zaumseil, J.; Chang, J.-F.; Ou, E. C. W.; Ho, P. K. H.; Sirringhaus, H.; Friend, R. H., General Observation of N-Type Field-Effect Behaviour in Organic Semiconductors. Nature 2005, 434, 194-199.

11. Caironi, M.; Bird, M.; Fazzi, D.; Chen, Z.; Di Pietro, R.; Newman, C.; Facchetti, A.; Sirringhaus, H., Very Low Degree of Energetic Disorder as the Origin of High Mobility in an N-Channel Polymer Semiconductor. Adv. Funct. Mater. 2011, 21, 3371-3381.

12. Chen, Z.; Bird, M.; Lemaur, V.; Radtke, G.; Cornil, J.; Heeney, M.; McCulloch, I.; Sirringhaus, H., Origin of the Different Transport Properties of Electron and Hole Polarons in an Ambipolar Polyselenophene-Based Conjugated Polymer. Phys. Rev. B 2011, 84, 115211 115211.

13. Khatib, O.; Mueller, A. S.; Stinson, H. T.; Yuen, J. D.; Heeger, A. J.; Basov, D. N., Electron and Hole Polaron Accumulation in Low-Bandgap Ambipolar Donor-Acceptor Polymer Transistors Imaged by Infrared Microscopy. Phys. Rev. B 2014, 90, 235307.

14. Takeda, N.; Asaoka, S.; Miller, J. R., Nature and Energies of Electrons and Holes in a Conjugated Polymer, Polyfluorene. J. Am. Chem. Soc. 2006, 128, 16073-16082.

15. Grozema, F. C.; Siebbeles, L. D. A.; Warman, J. M.; Seki, S.; Tagawa, S.; Scherf, U., Hole Conduction Along Molecular Wires: $\Sigma$-Bonded Silicon Versus П-Bond-Conjugated Carbon. Adv. Mat. 2002, 14, 228-231. 
16. Hoofman, R. J. O. M.; de Haas, M. P.; Siebbeles, L. D. A.; Warman, J. M., Highly Mobile Electrons and Holes on Isolated Chains of the Semiconducting Polymer Poly(Phenylene Vinylene). Nature 1998, 392, 54-56.

17. Grozema, F. C.; Warman, J. M., Highly Mobile Electrons and Holes on Polyfluorene Chains Formed by Charge Scavenging in Pulse-Irradiated Trans-Decalin Solutions. Radiat. Phys. Chem. 2005, 74, 234-238.

18. Cornil, J.; Gueli, I.; Dkhissi, A.; Sancho-Garcia, J. C.; Hennebicq, E.; Calbert, J. P.; Lemaur, V.; Beljonne, D.; Brédas, J. L., Electronic and Optical Properties of Polyfluorene and Fluorene-Based Copolymers: A Quantum-Chemical Characterization. J. Chem. Phys. 2003, 118, 6615-6623.

19. Van Vooren, A.; Kim, J.-S.; Cornil, J., Intrachain Versus Interchain Electron Transport in Poly(Fluorene-Alt-Benzothiadiazole): A Quantum-Chemical Insight. ChemPhysChem 2008, 9, 989-993.

20. Zaumseil, J.; Groves, C.; Winfield, J. M.; Greenham, N. C.; Sirringhaus, H., ElectronHole Recombination in Uniaxially Aligned Semiconducting Polymers. Adv. Funct. Mater. 2008, 18, 3630-3637.

21. Zhang, Y.; Blom, P. W. M., Electron and Hole Transport in Poly(FluoreneBenzothiadiazole). Appl. Phys. Lett. 2011, 98, 143504.

22. Tanase, C.; Meijer, E. J.; Blom, P. W. M.; de Leeuw, D. M., Unification of the Hole Transport in Polymeric Field-Effect Transistors and Light-Emitting Diodes. Phys. Rev. Lett. 2003, 91, 216601.

23. Naber, R. C. G.; Bird, M.; Sirringhaus, H., A Gate Dielectric That Enables High Ambipolar Mobilities in Polymer Light-Emitting Field-Effect Transistors. Appl. Phys. Lett. 2008, 93, 023301. 
24. Frisch, M. J.; Trucks, G. W.; H. B. Schlegel; G. E. Scuseria; M. A. Robb; J. R.

Cheeseman; G. Scalmani; V. Barone; B. Mennucci; G. A. Petersson, et al. Gaussian 09, Revision A.2, Gaussian, Inc.: Wallingford CT, 2009.

25. Pedersen, L.; Pedersen, S. U., Private Communication. U. University of Aarhus, 2005.

26. Pedersen, S. U.; Bo Christensen, T.; Thomasen, T.; Daasbjerg, K., New Methods for the Accurate Determination of Extinction and Diffusion Coefficients of Aromatic and Heteroaromatic Radical Anions in N,N-Dimethylformamide. J. Electroanal. Chem. 1998, 454, 123-143.

27. Jagur-Grodzinski, J.; Feld, M.; Yang, S. L.; Szwarc, M., Electron Affinities of Aromatic Hydrocarbons in Tetrahydrofuran Solution. J. Phys. Chem. 1965, 69, 628-635.

28. Campbell, A. J.; Bradley, D. D. C.; Antoniadis, H., Dispersive Electron Transport in an Electroluminescent Polyfluorene Copolymer Measured by the Current Integration Time-ofFlight Method. Appl. Phys. Lett. 2001, 79, 2133-2135.

29. Cardona, C. M.; Li, W.; Kaifer, A. E.; Stockdale, D.; Bazan, G. C., Electrochemical Considerations for Determining Absolute Frontier Orbital Energy Levels of Conjugated Polymers for Solar Cell Applications. Adv. Mat. 2011, 23, 2367-2371.

30. Campbell, A. J.; Bradley, D. D. C.; Antoniadis, H., Quantifying the Efficiency of Electrodes for Positive Carrier Injection into Poly(9,9-Dioctylfluorene) and Representative Copolymers. J. Appl. Phys. 2001, 89, 3343-3351.

31. Fenwick, O.; Fusco, S.; Baig, T. N.; Di Stasio, F.; Steckler, T. T.; Henriksson, P.; Fléchon, C.; Andersson, M. R.; Cacialli, F., Efficient Red Electroluminescence from Diketopyrrolopyrrole Copolymerised with a Polyfluorene. APL Mater. 2013, 1, 032108.

32. Montalti, M.; Credi, A.; Prodi, L.; Teresa Gandolfi, M., Handbook of Photochemistry, Third Edition. CRC Press: 2006.

33. Chi, C.; Im, C.; Wegner, G., Lifetime Determination of Fluorescence and Phosphorescence of a Series of Oligofluorenes. J. Chem. Phys. 2006, 124, 024907. 
34. Burrows, H. D.; Miguel, M. d. G.; Monkman, A. P.; Hamblett, I.; Navaratnam, S., Transient Absorption Spectra of Triplet States and Charge Carriers of Conjugated Polymers. J. Mol. Struct. 2001, 563-564, 41-50.

35. Maciejewski, A.; Jaworska-Augustyniak, A.; Szeluga, Z.; Wojtczak, J.; Karolczak, J., Determination of Ferrocene Triplet Lifetime by Measuring T1 $\rightarrow \mathrm{T} 1$ Energy Transfer to Phenylosazone-D-Glucose. Chem. Phys. Lett. 1988, 153, 227-232.

36. Herkstroeter, W. G., Triplet Energies of Azulene, .Beta.-Carotene, and Ferrocene. J. Am. Chem. Soc. 1975, 97, 4161-4167.

37. Shcheglo.Na; Shigorin, D. N.; Yakobson, G. G.; Tushishv.Ls, Effects of Halide Substituents on Position and Nature of Lower Electronic Levels in Certain Quinones. Russ. J. Phys. Chem. 1969, 43, 1112.

38. Darmanyan, A. P.; Foote, C. S., Effect of Sensitizer Heavy Atoms on Singlet Oxygen Generation Efficiency. J. Phys. Chem. 1992, 96, 3723-3728.

39. Huang, S. S. S.; Freeman, G. R., Electron Transport in Gaseous, Critical, and Liquid Benzene and Toluene. J. Chem. Phys. 1980, 72, 2849-2855.

40. Bird, M. J.; Reid, O. G.; Cook, A. R.; Asaoka, S.; Shibano, Y.; Imahori, H.; Rumbles, G.; Miller, J. R., Mobility of Holes in Oligo- and Polyfluorenes of Defined Lengths. J. Phys. Chem. C 2014, 118, 6100-6109.

41. Centineo, G.; Fragala, I.; Bruno, G.; Spampinato, S., Photoelectron Spectroscopy of Benzophenone, Acetophenone and Their Ortho-Alkyl Derivatives. J. Mol. Struct. 1978, 44, 203-210.

42. Lias, S. G., "Ion Energetics Data". In NIST Chemistry WebBook, NIST Standard Reference Database Number 69, Linstrom, P. J.; Mallard, W. G., Eds. National Institute of Standards and Technology, Gaithersburg MD, 20899, 2016. 
43. Cornil, J.; Beljonne, D.; Bredas, J. L., Nature of Optical-Transitions in Conjugated Oligomers .1. Theoretical Characterization of Neutral and Doped Oligo(Phenylenevinylene)S. J. Chem. Phys. 1995, 103, 834-841.

44. Deussen, M.; Bassler, H., Anion and Cation Absorption-Spectra of Conjugated Oligomers and Polymers. Chem. Phys. 1992, 164, 247-257.

45. Oberski, J. M.; Greiner, A.; Bassler, H., Absorption-Spectra of the Anions of Phenylenevinylene Oligomers and Polymer. Chem. Phys. Lett. 1991, 184, 391-397.

46. Mulliken, R. S., Molecular Compounds and Their Spectra .2. J. Am. Chem. Soc. 1952, 74, 811-824.

47. Hush, N. S., Homogeneous and Heterogeneous Optical and Thermal Electron Transfer. Electrochimica Acta 1968, 13, 1005-23.

48. Hush, N. S., Distance Dependence of Electron-Transfer Rates. Coord. Chem. Rev. 1985, 64, 135-157.

49. Creutz, C.; Newton, M. D.; Sutin, N., Metal-Ligand and Metal-Metal Coupling Elements. J. Photochem. Photobiol., A 1994, 82, 47-59.

50. Brunschwig, B. S.; Creutz, C.; Sutin, N., Optical Transitions of Symmetrical MixedValence Systems in the Class Ii-Iii Transition Regime. Chem. Soc. Rev. 2002, 31, 168-184.

51. Jespersen, K. G.; Beenken, W. J. D.; Zaushitsyn, Y.; Yartsev, A.; Andersson, M.; Pullerits, T.; Sundstrom, V., The Electronic States of Polyfluorene Copolymers with Alternating Donor-Acceptor Units. J. Chem. Phys. 2004, 121, 12613-12617.

52. Slates, R. V.; Szwarc, M., Dissociative Equilibria in Systems Aromatic HydrocarbonNa+-]-[ Radical Anion- + Na+. J. Phys. Chem. 1965, 69, 4124-4131.

53. Bradley, D. D. C.; Grell, M.; Long, X.; Mellor, H.; Grice, A. W.; Inbasekaran, M.; Woo, E. P. In Influence of Aggregation on the Optical Properties of a Polyfluorene, SPIE San Diego, CA, United States, San Diego, CA, United States, 1997; pp 254-259. 
54. Grell, M.; Bradley, D. D. C.; Ungar, G.; Hill, J.; Whitehead, K. S., Interplay of Physical Structure and Photophysics for a Liquid Crystalline Polyfluorene. Macromolecules 1999, 32, 5810-5817.

55. Badger, B.; Brocklehurst, B.; Russell, R. D., The Naphthalene Dimer Cation, (C10h8)(2)(+). Chem. Phys. Lett. 1967, 1, 122-124.

56. Kira, A.; Arai, S.; Imamura, M., Pyrene Dimer Cation as Studied by Pulse Radiolysis. J. Chem. Phys. 1971, 54, 4890.

57. Meotner, M.; Hamlet, P.; Hunter, E. P.; Field, F. H., Bonding Energies in Association Ions of Aromatic-Compounds - Correlations with Ionization Energies. J. Am. Chem. Soc. 1978, $100,5466-5471$.

58. Kira, A.; Imamura, M., Absorption-Spectra of Dimer Cations and Other Cationic Species Produced by Warming of Gamma-Irradiated Glassy Solutions of AromaticHydrocarbons. . J. Phys. Chem. 1979, 83, 2267-2273.

59. Meotner, M., Dimer Cations of Polycyclic Aromatics - Experimental Bonding Energies and Resonance Stabilization. . J. Phys. Chem. 1980, 84, 2724-2728.

60. Liem, H. M.; Etchegoin, P.; Whitehead, K. S.; Bradley, D. D. C., Raman Anisotropy Measurements: An Effective Probe of Molecular Orientation in Conjugated Polymer Thin Films. Adv. Funct. Mater. 2003, 13, 66-72.

61. Bright, D. W.; Dias, F. B.; Galbrecht, F.; Scherf, U.; Monkman, A. P., The Influence of Alkyl-Chain Length on Beta-Phase Formation in Polyfluorenes. Adv. Funct. Mater. 2009, 19, 67-73.

62. Sato, M.; Kumada, A.; Hidaka, K.; Hirano, T.; Sato, F., Computational Study of Excess Electron Mobility in High-Pressure Liquid Benzene. J. Phys. Chem. C 2016, 120, 8490-8501.

63. Itoh, K.; Holroyd, R., Effect of Pressure on the Electron Mobility in Liquid Benzene and Toluene. J. Phys. Chem. 1990, 94, 8850-8854. 
64. Yang, C.-M.; Liao, H.-H.; Horng, S.-F.; Meng, H.-F.; Tseng, S.-R.; Hsu, C.-S., Electron Mobility and Electroluminescence Efficiency of Blue Conjugated Polymers. Synthetic Metals 2008, 158, 25-28.

65. Zaumseil, J.; Donley, C. L.; Kim, J. S.; Friend, R. H.; Sirringhaus, H., Efficient TopGate, Ambipolar, Light-Emitting Field-Effect Transistors Based on a Green-Light-Emitting Polyfluorene. Adv. Mat. 2006, 18, 2708-2712.

66. Redecker, M.; Bradley, D. D. C.; Inbasekaran, M.; Woo, E. P., Nondispersive Hole Transport in an Electroluminescent Polyfluorene. Appl. Phys. Lett. 1998, 73, 1565-1567.

67. Arif, M.; Yun, M.; Gangopadhyay, S.; Ghosh, K.; Fadiga, L.; Galbrecht, F.; Scherf, U.; Guha, S., Polyfluorene as a Model System for Space-Charge-Limited Conduction. Phys. Rev. B 2007, 75, 195202.

68. Lee, W. K.; Liu, B.; Park, C. W.; Shine, H. J.; Guzman-Jimenez, I. Y.; Whitmire, K. H., Addition of Thianthrene Cation Radical to Cycloalkenes. An Unexpected Monoadduct. J. Org. Chem. 1999, 64, 9206-9210.

69. Schins, J. M.; Prins, P.; Grozema, F. C.; Abellon, R. D.; de Haas, M. P.; Siebbeles, L. D. A., Development of a Microwave Transmission Setup for Time-Resolved Measurements of the Transient Complex Conductivity in Bulk Samples. Rev. Sci. Instrum. 2005, 76, 084703 $1-9$.

70. Infelta, P. P.; de Haas, M. P.; Warman, J. M., The Study of the Transient Conductivity of Pulse Irradiated Dielectric Liquids on a Nanosecond Timescale Using Microwaves. Radiat. Phys. Chem. 1977, 10, 353-365.

71. Warman, J. M.; Infelta, P. P.; De Haas, M. P.; Hummel, A., The Study of Primary and Secondary Charge Carriers in Nanosecond Pulse Irradiated Liquid Dielectrics Using a Resonant Microwave Cavity. Can. J. Chem. 1977, 55, 2249-2257.

72. Huang, S. S.-S.; Freeman, G. R., Cation Transport in Gaseous, Critical, and Liquid Benzene and Toluene. J. Chem. Phys. 1980, 72, 1989-1993. 
73. Li, X.; Bird, M.; Mauro, G.; Asaoka, S.; Cook, A. R.; Chen, H. C.; Miller, J. R., Transport of Triplet Excitons Along Continuous 100 Nm Polyfluorene Chains. J. Phys. Chem. B 2015, 119, 7210-7218.

74. Wishart, J. F.; Cook, A. R.; Miller, J. R., The Leaf Picosecond Pulse Radiolysis

Facility at Brookhaven National Laboratory. Rev. Sci. Instrum. 2004, 75, 4359-4366. 
TABLE OF CONTENTS GRAPHIC






\title{
Supporting Information
}

\section{Fast Holes, Slow Electrons and Medium Control of Polaron Size and Mobility in the DA Polymer F8BT}

\author{
Matthew J. Bird, ${ }^{*}$ Jin Bakalis, Sadayuki Asaoka, Henning Sirringhaus, and John R. Miller

\section{Obtaining real and imaginary conductivity with PR-TRMC}

The procedure for finding the real and imaginary conductivity is as follows. Firstly transmission line theory ${ }^{1}$ is used to simultaneously fit the reflected power vs frequency dips for both the empty cavity and the filled cavity (figure S1a). The reflected power is calculated by converting the voltage measured by the detector using a calibration curve. During this fitting procedure, the cavity length, iris inductance, solution complex dielectric constant, and wall conductivity are allowed to vary. Due to an inherently imperfect circulator and a long distance $\sim 1.5 \mathrm{~m}$ from the circulator to the cavity, there are interference modulations in reflected power with a period, in frequency space, of $\sim 70 \mathrm{MHz}$. These modulations are included in the fitting model by introducing a 1-2\% voltage reflection coefficient $\sim 1.5 \mathrm{~m}$ in front of the cavity and the exact distance and reflection coefficient are also fitting parameters in the model. The cavity length had been chosen so that the empty and filled cavity had resonances at a similar frequency, although they correspond to the $\mathrm{TE}_{101}$ and $\mathrm{TE}_{102}$ modes respectively.

After the steady state reflected power dips have been fitted, all the parameters are held constant apart from the dielectric properties of the filled cavity. Using 30-40 transient absorption traces like those in figure S1b, new reflected power dips are constructed at various times following the electron pulse and are fitted to the transmission line model. Figure S1c shows the changes to the reflected power at 100 ns after the start of the electron pulse and the global fit to all the data. By repeating this process at various times, the time-dependent complex dielectric constant of the cavity can be recovered and plotted as the real and imaginary parts of the conductivity as shown in figure S1d, following the relationships outlined below.

The electric field for the propagating microwaves has terms with $e^{i(k z-\omega t)}$, where the propagation constant, $k$, is given by:

$$
k^{2}=\mu_{0} \varepsilon_{0} \varepsilon_{r} \omega^{2}+i \mu_{0} \sigma \omega
$$

$z$ is the direction of propagation, $\sigma$ is the conductivity in the waveguide, $\omega$ is the angular frequency of the microwaves, and all other symbols have their usual meaning. In some books, the propagation constant, $\gamma=\boldsymbol{i} k$, and the engineer's convention for the exponential, $e^{\boldsymbol{i} \omega t-\gamma z}$, will be used. Here, following the physicist's convention, the complex dielectric constant and conductivity are written as:

$$
\begin{aligned}
\boldsymbol{\varepsilon}_{\boldsymbol{r}} & =\varepsilon^{\prime}{ }_{r}+\boldsymbol{i} \varepsilon^{\prime \prime}{ }_{r} \\
\boldsymbol{\sigma} & =\sigma^{\prime}+\boldsymbol{i} \sigma^{\prime \prime}
\end{aligned}
$$

From the above equations, the real and imaginary part of the conductivity can be shown to be related complex dielectric constant by:

$$
\sigma^{\prime}=\varepsilon^{\prime \prime}{ }_{r} \varepsilon_{0} \omega
$$




$$
\sigma^{\prime \prime}=\varepsilon_{0} \omega\left(1-\varepsilon_{r}^{\prime}\right)
$$

and these last two relationships hold for both the engineer's and physicist's conventions.
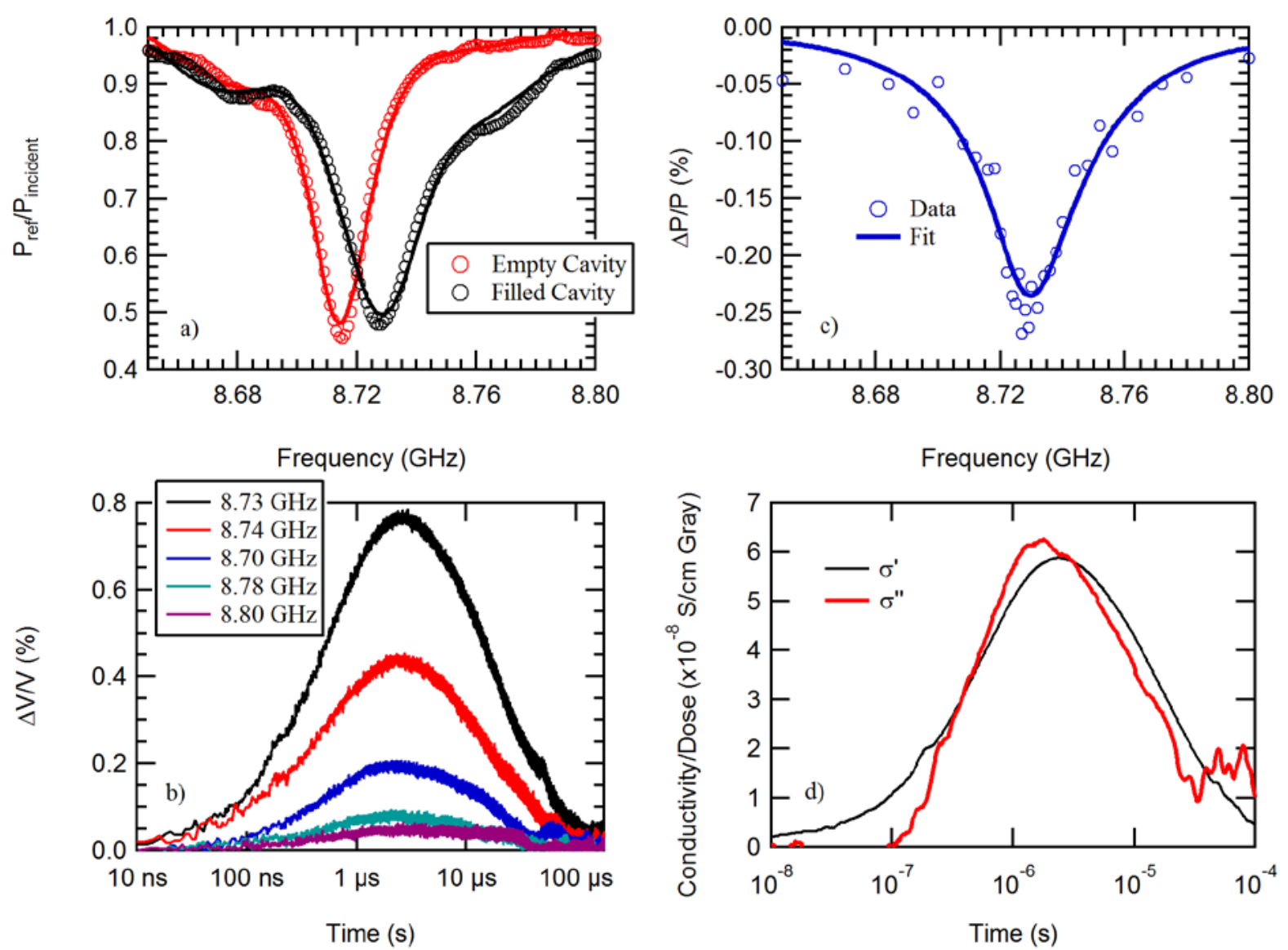

Figure S1 a) Experimental data (markers) and fits (lines) to the normalized reflected power from the microwave cavity when empty and filled with F8BT (0.5mM PRU) + Biphenyl $(5 \mathrm{mM})$ in Ar-saturated benzene. b) Examples of transient microwave absorption traces, as a \% change in detector voltage, at various frequencies following a $200 \mathrm{~ns} 2 \mathrm{MeV}$ electron pulse for the same solution as in a). c) Percentage change in microwave power at 100 ns at various frequencies using transients including those in b). d) Real and imaginary parts of the conductivity following the full global analysis of transient absorption at all frequencies measured (same solution as in Figure a-c).

\section{Using a PEDOT gate electrode to minimize electrostriction effects during CMS measurements}

After initially seeing device-to-device variations in F8BT polaron spectra, simple tests were performed on various transistor and capacitor (MIS) structures with/without F8BT present (see Figure S2). These tests showed that there were three different origins of changes in transmission upon modulation of the voltage: electroabsorption, electrostriction and polaron absorption/neutral bleaching. The effects of electrostriction and electroabsorption were most prominent in the capacitor structure and largely appear in phase with applied voltage. A component of the polaron absorption spectrum could be distinguished as a positive change in transmission in the out-of-phase channel in the transistor structures due to the phase lag between the gate voltage and the charge density in the channel (the other two effects being instantaneous with field at the modulation frequencies used here). The exact sign of the 
change in transmission depends on whether the device is in hole accumulation or electron accumulation as the lock-in reference signal from the signal generator is independent of the DC component of the voltage output so effectively changes by 180 degrees relative to the phase of the charge absorption when going from hole to electron accumulation.
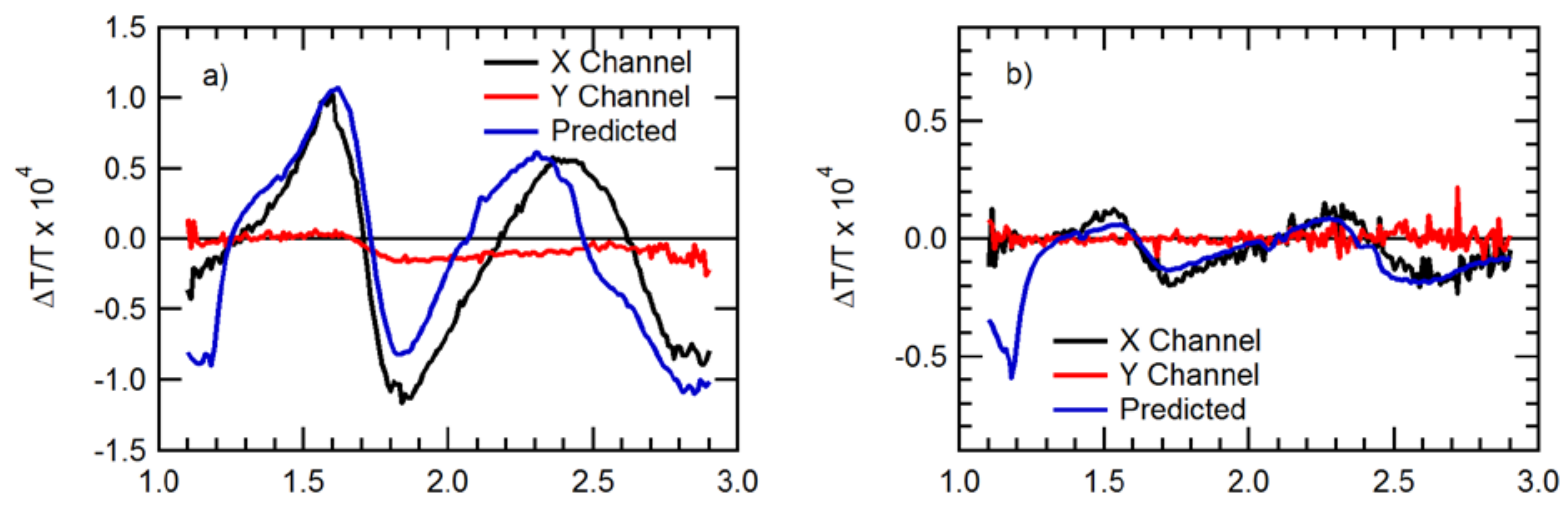

Energy (eV)
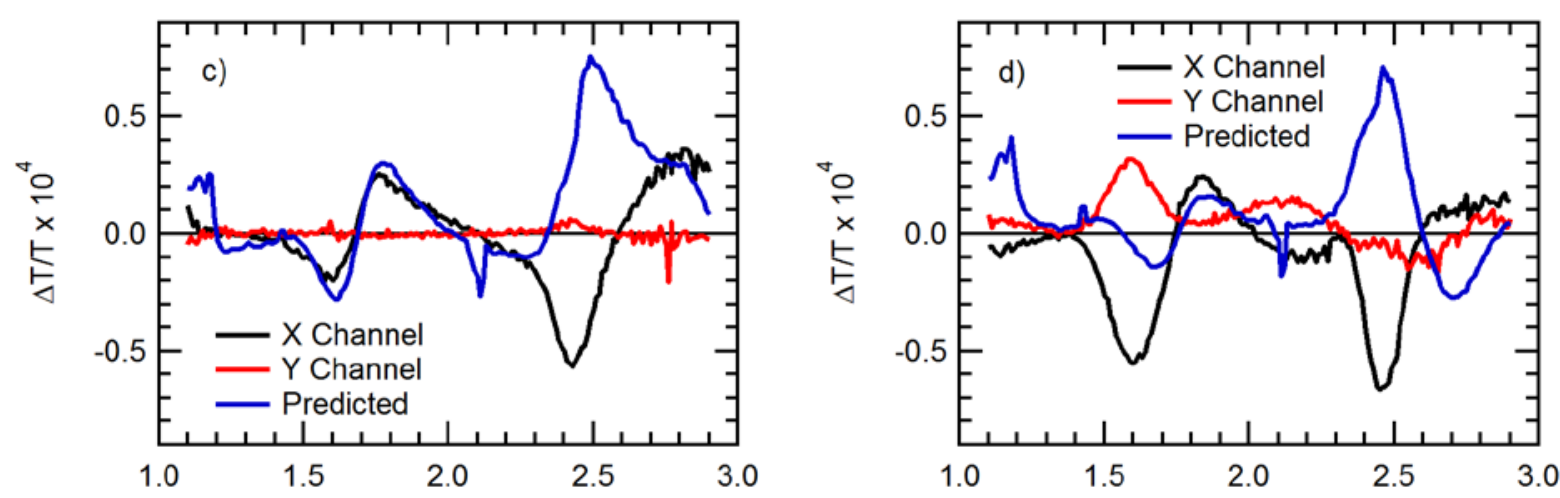

Energy (eV)

Energy $(\mathrm{eV})$

Figure S2. Experimentally measured changes in transmission through various device structures measured with a lock-in amplifier (locked to the $37 \mathrm{~Hz}$ gate voltage modulation) with both the in-phase (X Channel) and out-of-phase (Y Channel) signals displayed. Also shown is the predicted change in transmission due to the electrostriction effect caused by variations in device thickness with electric field. Device are: a) Au(20 nm) / PMMA (470 nm) / Al (6nm) parallel plate capacitor structure $\mathrm{V}_{\mathrm{g}}=+50 \mathrm{~V}, \mathrm{~V}_{\mathrm{pp}}=10 \mathrm{~V}$, b) Au (20 nm) / PMMA $(470 \mathrm{~nm}) / \mathrm{Al}(6 \mathrm{~nm})$ transistor structure $\left.\mathrm{V}_{\mathrm{g}}=+30 \mathrm{~V}, \mathrm{~V}_{\mathrm{pp}}=10 \mathrm{~V}, \mathrm{c}\right) \mathrm{Au}(20 \mathrm{~nm}) / \mathrm{F} 8 \mathrm{BT}(30$ $\mathrm{nm}) /$ PMMA (470 nm) / $\mathrm{Al}(6 \mathrm{~nm})$ parallel plate capacitor structure $\mathrm{V}_{\mathrm{g}}=-50 \mathrm{~V}, \mathrm{~V}_{\mathrm{pp}}=10 \mathrm{~V}$, d) $\mathrm{Au}(20 \mathrm{~nm}) / \mathrm{F} 8 \mathrm{BT}(30 \mathrm{~nm}) / \operatorname{PMMA}(470 \mathrm{~nm}) / \mathrm{Al}(6 \mathrm{~nm}) \mathrm{V}_{\mathrm{g}}=-50 \mathrm{~V}, \mathrm{~V}_{\mathrm{pp}}=10 \mathrm{~V}$ transistor structure. The transistor structures were bottom contact/top gate and had interdigitated source-drain electrodes with channel length $40 \mu \mathrm{m}$, width $2 \mathrm{~cm}$ and an approximate ratio of electrode to channel area of 1:1.

It can be shown ${ }^{2}$ that changes in transmission caused by electrostriction can be modelled by the following equation based on the equation for transmission through a Fabry Perot etalon and the response to a force between two parallel charge sheets:

$$
\frac{d T}{T}=\frac{\pi n F \sin \left(\frac{4 \pi n l}{\lambda}\right)}{Y \lambda\left(1+\sin ^{2}\left(\frac{2 \pi n l}{\lambda}\right)\right)}\left(\frac{\varepsilon_{0} \varepsilon_{r} V_{p p}\left|V_{g}\right|}{l}+\sigma V_{p p}\right)
$$


where $n$ is the refractive index, $Y$ is the Young's modulus, $F$ is the Finesse of the optical cavity, $V_{p p}$ is the peak to peak voltage modulation, $V_{g}$ is the gate voltage, $l$ is the distance between the coplanar electrodes, $\sigma$ is any trapped charge density that is creating a steady state electric field between the electrodes and other symbols have their usual meaning. It can also be shown ${ }^{2}$ that the spectrum obtained from the CMS experiment can be related to the derivative of the steady state transmission through the device ( $T$ vs photon energy, $E$ ) according to the following equation:

$$
\left(\frac{d T}{T}\right)_{E, n}=-\frac{1}{T}\left(\frac{d T}{d E}\right)_{l, n}\left(\frac{\varepsilon_{0} \varepsilon_{r} V_{p p}\left|V_{g}\right| E}{2 l^{2} Y}\right)
$$

Figure S2 confirms the electrostriction model by comparing the predicted spectrum, based on the derivative of the steady state transmission, to the measured one. Changes to the dielectric constant due to the applied field cannot be used to explain the observed changes in transmission as the sign would be opposite. The electrostriction model fails to reproduce the observed changes in transmission in the region where electroabsorption occurs as can be seen in Figure S2 at energies above $2.4 \mathrm{eV}$ when F8BT is present, although this also resembles the derivative of the transmission spectra, but with a different sign.

To minimize the electrostriction (and electroabsorption) artifacts, thick source and drain electrodes were used to block light from the region where these effects are strongest. To minimize the electrostriction effects in the channel area, the shiny Al gate electrode was replaced with PEDOT which was patterned to just cover the transistor area. There were indications that there were features in the CMS spectrum originating from modulating the density of carriers in the PEDOT gate electrode. This was clarified by making transistors with aligned F8BT, but where the PEDOT is isotropic. By measuring the spectrum with polarized light (Figure S3a and S3b), it was possible to remove the absorption from the F8BT polarons and just record the changes in the PEDOT cation spectrum, which, in the region measured here, appears as a bleach due to the way the PEDOT spectrum evolves with doping. ${ }^{3}$ The extracted PEDOT contribution is shown in Figure S3c. 

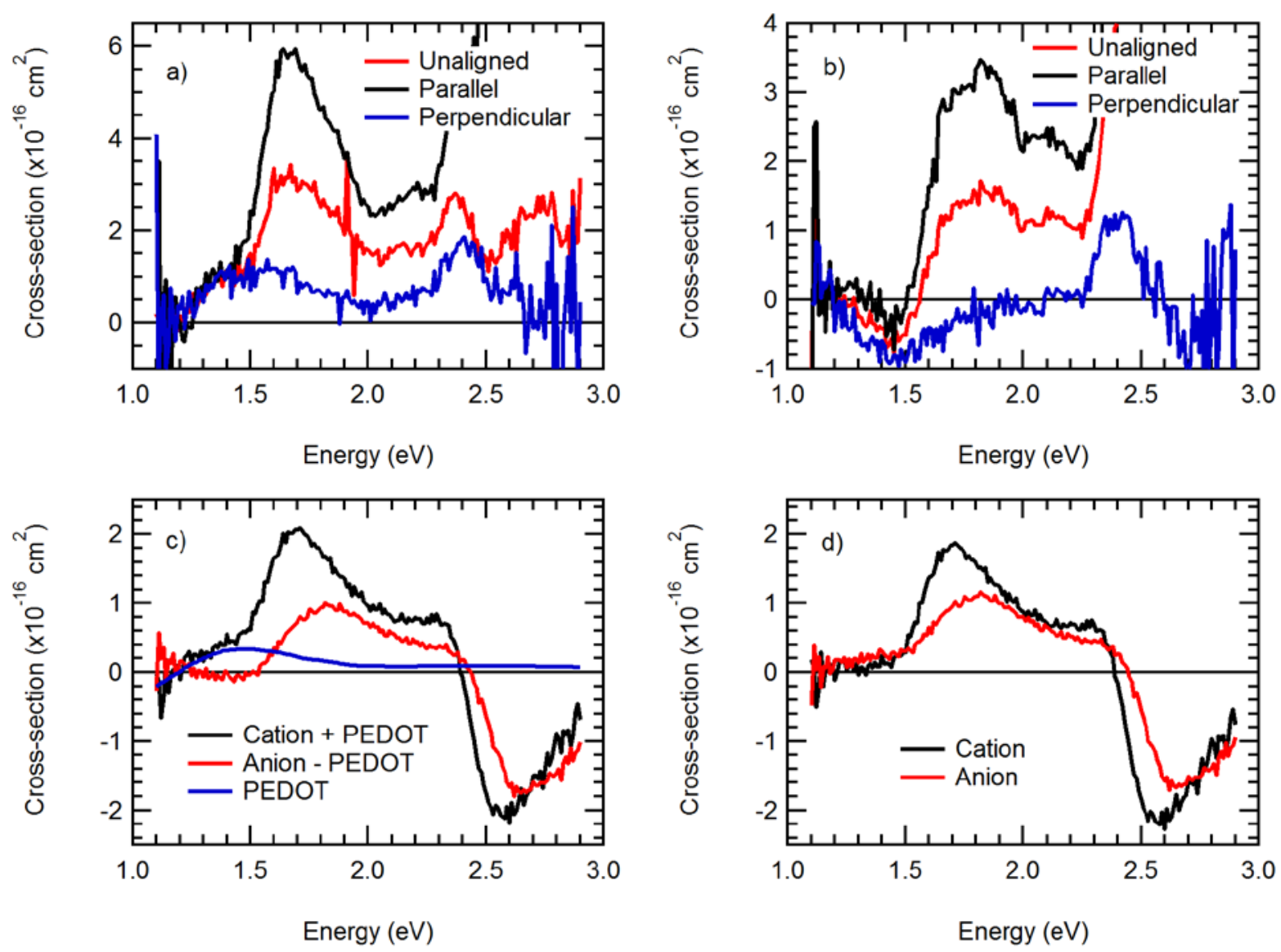

Figure S3. a) Cross section for F8BT hole absorption measured with CMS on bottom contact, top gate OFET device: Al (17nm) / Au (20nm) / F8BT (aligned) (60nm) / PMMA (750nm) / PEDOT:PSS. b) Same as in a) except for being the absorption of electrons. c) Absorption spectra of holes and electrons on F8BT with a PEDOT gate on a device optimized for highest signal to noise and with a $5 \mathrm{~V}$ drain bias. Spectrum is obtained from the out of phase channel of the lock-in to remove electro-absorption and is scaled to the correct magnitude based on measurements taken at $1.69 \mathrm{eV}$, away from the electroabsorption region using the total magnitude of the absorption (including both in phase and out of phase components). d) Final spectra of hole and electrons on F8BT, corrected for both electrostriction and electroabsorption and with the correct magnitude of cross-section.

To get the final polaron spectra, devices with unaligned F8BT and PEDOT gate electrodes were used and the known PEDOT spectrum was subtracted. The reason for this was that the aligned F8BT devices show a much larger contact resistance, due to the required thicker F8BT layer, leading to larger electroabsorption effects as the charges cannot move in fast enough to screen the F8BT from the gate field. Once the cross-section had been measured accurately at a particular wavelength, the full spectrum was taken with a $5 \mathrm{~V}$ drain bias applied which improved the signal-to-noise ratio. This can be explained by the superlinear increase in current observed in source-drain current at low drain voltages that is often attributed to contact resistance; the $5 \mathrm{~V}$ drain voltage allowed faster charging of the accumulation layer.

\section{Determining charge density for determination of mobility}

Mobility, $\mu\left(\mathrm{cm}^{2} / \mathrm{Vs}\right)$, is related to conductivity, $\sigma(\mathrm{S} / \mathrm{cm})$, by the following equation:

$$
\mu=\frac{\sigma}{N e}
$$


where $N$ is the density $\left(\mathrm{cm}^{-3}\right)$ of charges and $e$ is the charge on an electron. In order to know the mobility, the charge density must be known. In Figure 5b, it was seen that the measured conductivity from the electron and hole, individually, for polyfluorene did not add up to the conductivity without scavengers. In theory, there should be an equal concentration of electrons and holes, but this was tested with pulse radiolysis transient absorption spectroscopy on the same solutions after they had been taken out of the waveguide. Figure S4 shows the transient optical absorption at $580 \mathrm{~nm}$ and $760 \mathrm{~nm}$ for the three polyfluorene solutions. At 580 $\mathrm{nm}$, both the electron and hole absorb with similar extinction coefficients: $60,000 \mathrm{M}^{-1} \mathrm{~cm}^{-1}$ and $56,500 \mathrm{M}^{-1} \mathrm{~cm}^{-1}$ respectively from Table 1 , adjusted to $580 \mathrm{~nm}$.. There is a small contribution at $580 \mathrm{~nm}$ from the triplet absorption, which peaks at $760 \mathrm{~nm}$, but, as shown in S4b, the triplets have vanished by $2 \mu \mathrm{s}$, so it can be seen that there were more holes than electrons in these solutions.
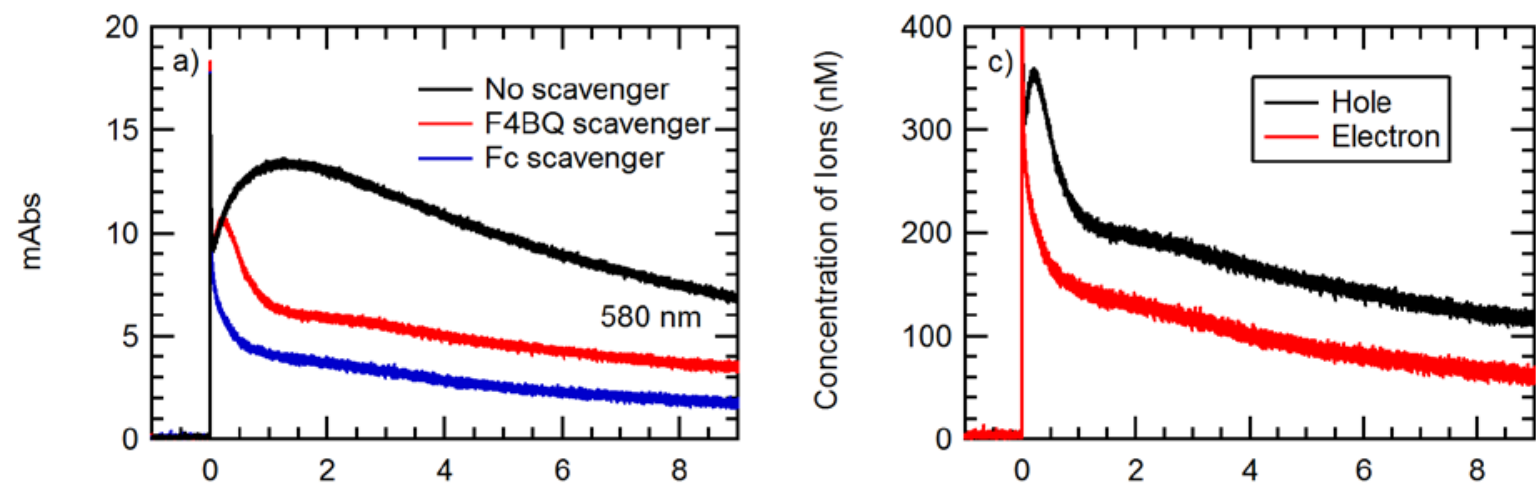

Time $(\mu \mathrm{s})$

Time $(\mu \mathrm{s})$
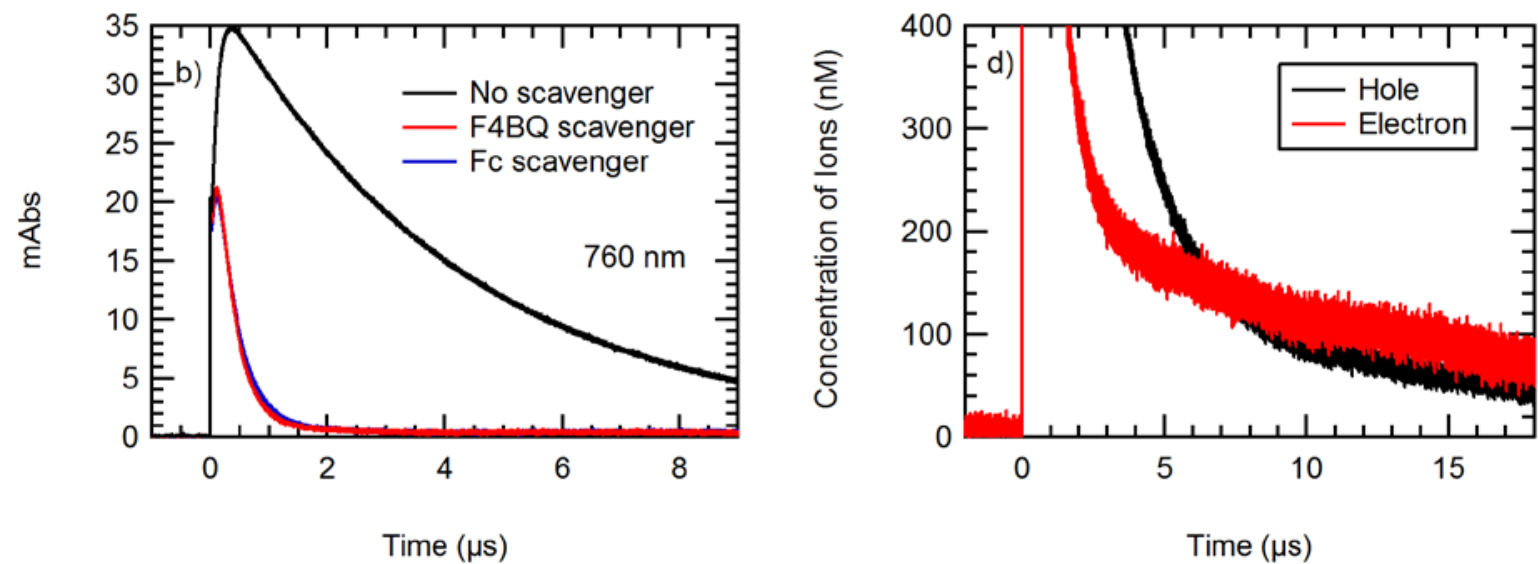

Figure S4. Pulse Radiolysis transient absorption traces at a) $580 \mathrm{~nm}$ (polarons) and b) $760 \mathrm{~nm}$ (triplets) of solutions of polyfluorene $(0.5 \mathrm{mM}$ PRU) used in the microwave conductivity experiment with either: no scavenger (black), $0.5 \mathrm{mM}$ F4BQ (red), or $0.5 \mathrm{mM}$ Ferrocene (blue). All solutions were oxygen free and also contained $5 \mathrm{mM}$ biphenyl. Absorption data from PR-TAS measurements converted into concentration of ions for the c) polyfluorene solutions and d) F8BT solutions for doses of 35 Gray and 26 Gray (J/kg) respectively.

The feature in the red trace in Figure S4a that decays by $2 \mu \mathrm{s}$ is believed to be the state that is formed when the $\mathrm{pF}$ triplet undergoes electron transfer to the F4BQ; assigning it to the triplet can be ruled out by a comparison to Figure S4b and the fact that the absorption is also seen at $2151 \mathrm{~nm}$ where charges, but not triplets, absorb. The exact nature of this state and whether there was partial or complete charge transfer is unknown, but it is clear, by comparison with 
the conductivity data, that it does not contain a charge that is free to move along the $\mathrm{pF}$ chain and give rise to microwave absorption.

Measured extinction coefficients in benzene, reported in the main manuscript, are used to turn the transient absorption traces into concentrations of pF and F8BT ion as shown in Figure S4c and S4d respectively. The concentration traces are only valid in the time region where only the ions are absorbing, which is dependent on overlapping absorptions from triplet species and how long they live for. The valid regions are as follows: $2 \mu$ s onwards for both $\mathrm{pF}$ ions, 3 $\mu$ s onwards for F8BT anions, and $10 \mu$ s onwards for F8BT cations due to the apparently slow reaction between F4BQ and F8BT triplets which absorb at the same wavelength. These concentration plots confirm the lower yield per unit dose of $\mathrm{pF}$ anions and are used in the final calculations of mobility.
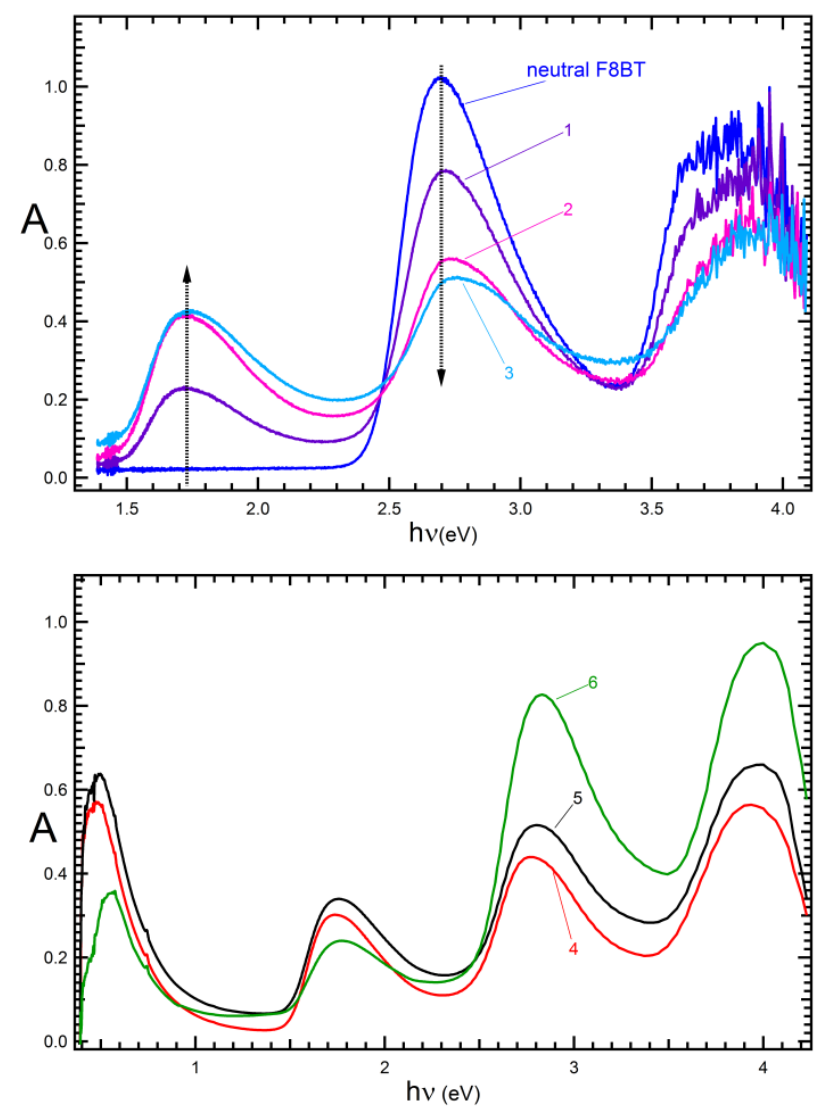

Figure S5. A series of the UV-Visible-NIR spectra of neutral F8BT (2.7 and $3.8 \mathrm{eV})$ and oxidized species $(0.5,1.7,2.8$, and $4 \mathrm{eV}$ ). $0.34 \mathrm{mM}$ (PRU) of F8BT solution in orthodichlorobenzene (ODCB) was chemically doped with nitrosonium hexafluorophosphate $\left(\mathrm{NO}^{+} \mathrm{PF}_{6}{ }^{-}\right)$. While $\mathrm{NOPF}_{6}$ did not noticeably dissolve in ODCB, oxidative doping did proceed using solid $\mathrm{NOPF}_{6}$ at the bottom of the vessel, aided by stirring. The labels indicate the sequence of doping. $\mathrm{NO}^{+}$ions have a redox potential of $1.4 \mathrm{eV}$ vs. SCE, approximately $0.6 \mathrm{eV}$ higher than that of $\mathrm{THI}^{++}$. They are expected to yield more extensive evolution of oxidized species upon F8BT doping. Early stages were recored by a spectrophotometer inside the glove box having a range only to $900 \mathrm{~nm}$ (upper panel). The sample then transferred to a spectrophotometer with a larger range (lower panel).

In Figure S1, the spectrum of pure neutral F8BT decreases with a blue-shift and develops a new transition at the wavelength close to neutral absorption with increasing doping levels. 
This suggests a possibility of an additional P3 band for $\mathrm{F} 8 \mathrm{BT}{ }^{\circ+}$ polarons in the region where the neutrals absorb. The calculation predicts $\mathrm{F}_{\mathrm{BBT}}{ }^{++}$polarons have a strong transition at the similar wavelength as the neutrals which supports the idea of P3.
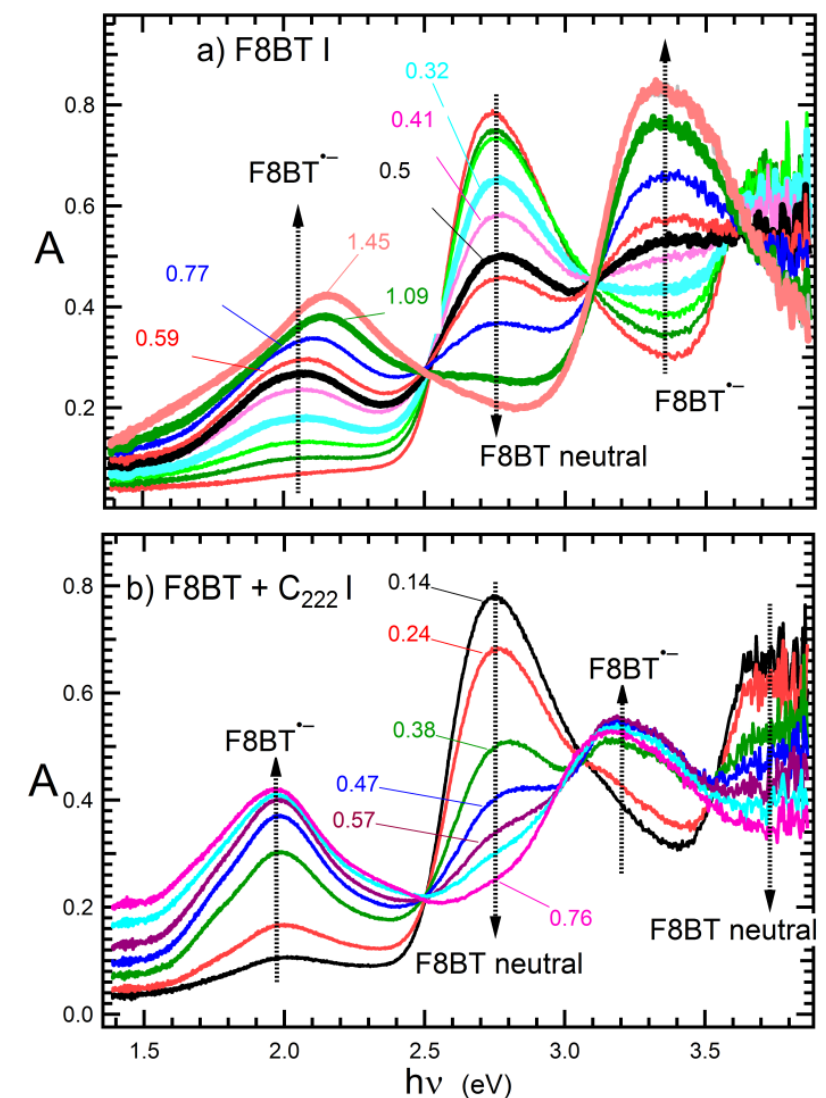

Figure S6. A series of the UV-Visible spectra of $0.3 \mathrm{mM}$ (PRU) F8BT in THF solution chemically doped with $\mathrm{Na}^{+} \mathrm{Bip}^{\bullet-}$ a) reduction I in the absence of $\mathrm{C}_{222}$ b) reduction $\mathrm{I}$ in the presence of $18 \mathrm{mM}$ of $\mathrm{C}_{222}$. The labels are concentrations as Bip ${ }^{\bullet-} / \mathrm{PRU}$.This data utilized a spectrophotometer with a range extending to $900 \mathrm{~nm}(1.38 \mathrm{eV})$. This Figure compares the evolution of $\mathrm{F}_{8 \mathrm{BT}}{ }^{-}$in the absence and presence of $\mathrm{C}_{222}$. In the absence of $\mathrm{C}_{222}$ shown in a), the F8BT $^{*-}$ anion band at $1.99 \mathrm{eV}$ increases with very little shift up to $\mathrm{n} \sim 0.5 \mathrm{Bip}{ }^{-} / \mathrm{PRU}$, followed by a blue shift with further reductions. In the presence of $\mathrm{C}_{222} \mathrm{~b}$ ), the same band grows with very little spectral shift.

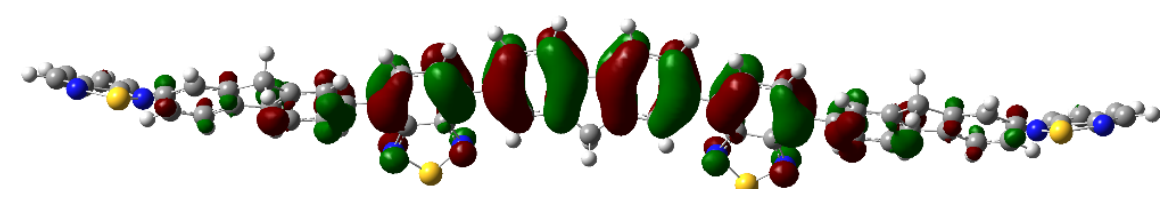

Figure S7. A isodensity plot of hole KS orbitals ( $\beta$ LUMO) of the cation of $\mathrm{F}_{4} \mathrm{BT}_{3}$ optimized by $\omega \mathrm{PBE}(\omega=0.1) / 3-21 \mathrm{~g}$ in chlorobenzene (PCM). 

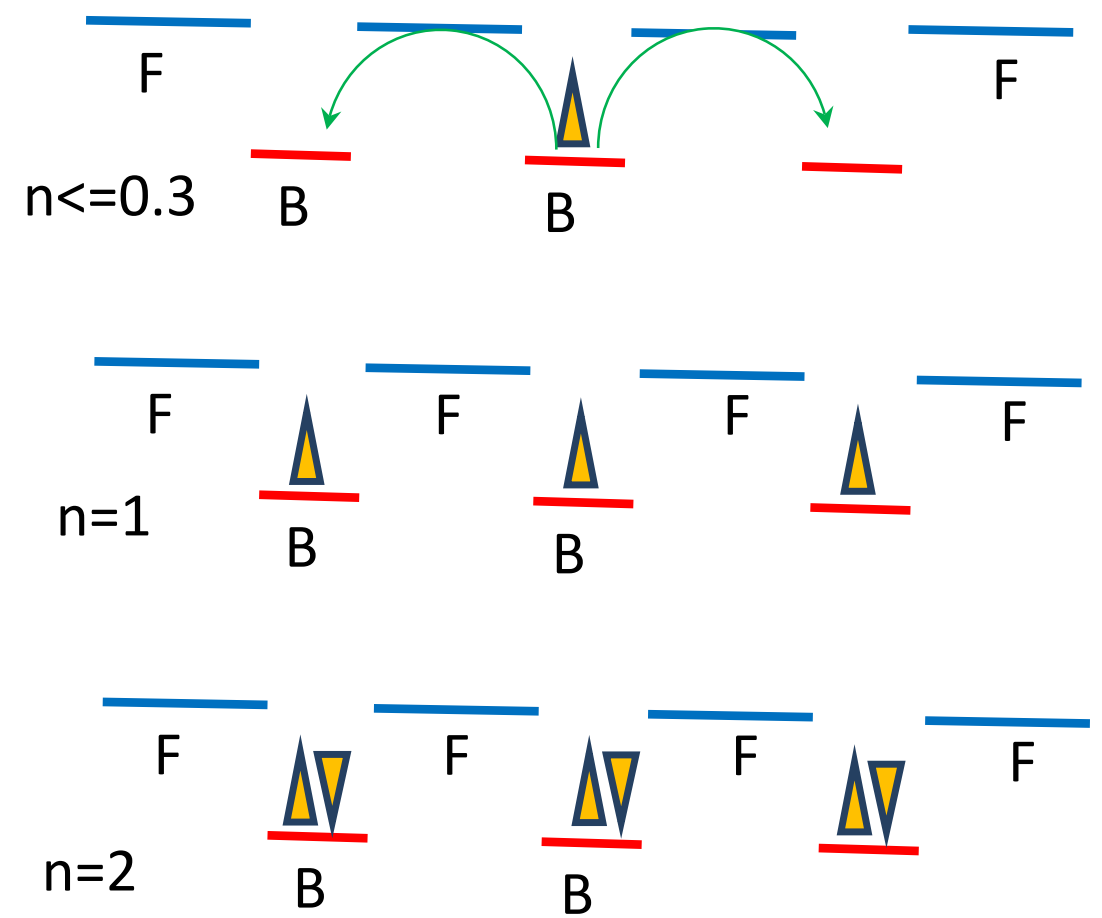

Figure S8. The effect of occupancy of benzothiadiazole (BT) and fluorene (F) units by electrons that reside on a single unit. At low occupancy ( $\mathrm{n}<0.3$ electrons/repeat unit) and electron (triangle) on the central BT unit can transfer to nearby, empty BT units. For $n=1$ every BT unit is occupied so no such transitions are possible. 




Figure S9. Kohn-Sham orbitals for a $\mathrm{BT}_{3} \mathrm{~F}_{4}$ oligomer containing 3 added electrons. The HOMO (278) and the two orbitals below it accommodate the three added electrons on BT groups. TDTFT computes no excited states lower than $1.59 \mathrm{eV}$. A strong band at $1.87 \mathrm{eV}$ transitions electrons to LUMOs 279, 280 and others, which have density on F and BT groups. A similar transition occurs at $1.76 \mathrm{eV}$ moves slightly more density to $\mathrm{F}$ groups.

Full reference to Gaussian software ${ }^{4}$

\section{REFERENCES}

1. Schins, J. M.; Prins, P.; Grozema, F. C.; Abellon, R. D.; de Haas, M. P.; Siebbeles, L. D. A., Development of a Microwave Transmission Setup for Time-Resolved Measurements of the Transient Complex Conductivity in Bulk Samples. Rev. Sci. Instrum. 2005, 76, 084703 $1-9$.

2. Bird, M. Optoelectronic Processes in Polyfluorene Ambipolar

Transistors. University of Cambridge, Cambridge, UK, 2010.

3. Baba, A.; Lübben, J.; Tamada, K.; Knoll, W., Optical Properties of Ultrathin Poly(3,4Ethylenedioxythiophene) Films at Several Doping Levels Studied by in Situ Electrochemical Surface Plasmon Resonance Spectroscopy. Langmuir 2003, 19, 9058-9064.

4. Frisch, M. J.; Trucks, G. W.; H. B. Schlegel; G. E. Scuseria; M. A. Robb; J. R. Cheeseman; G. Scalmani; V. Barone; B. Mennucci; G. A. Petersson; H. Nakatsuji; M. Caricato; X. Li, H. P. H.; A. F. Izmaylov; J. Bloino; G. Zheng; J. L. Sonnenberg; M. Hada; M. Ehara; K. Toyota; R. Fukuda; J. Hasegawa; M. Ishida; T. Nakajima; Y. Honda; O. Kitao; H. Nakai; T. Vreven; J. A. Montgomery, J.; J. E. Peralta; F. Ogliaro; M. Bearpark; J. J. Heyd; E. Brothers; K. N. Kudin; V. N. Staroverov; R. Kobayashi; J. Normand; K. Raghavachari; A. Rendell; J. C. Burant; S. S. Iyengar; J. Tomasi; M. Cossi; N. Rega; J. M. Millam; M. Klene; J. E. Knox; J. B. Cross; V. Bakken; C. Adamo; J. Jaramillo; R. Gomperts; R. E. Stratmann; O. Yazyev; A. J. Austin; R. Cammi; C. Pomelli; J. W. Ochterski; R. L. Martin; K. Morokuma; V. G. Zakrzewski; G. A. Voth; P. Salvador; J. J. Dannenberg; S. Dapprich; A. D. Daniels; Ö. Farkas; J. B. Foresman; J. V. Ortiz; J. Cioslowski; Fox, D. J. Gaussian 09, Revision A.2, Gaussian, Inc.: Wallingford CT, 2009. 